\title{
Phase Transitions in Three-Dimensional Bosonic Systems in Optical Lattices
}

\author{
H. Kleinert ${ }^{a}$ Z Z. Narzikulov ${ }^{b}$ and Abdulla Rakhimov ${ }^{a}$, 对 \\ a Institut für Theoretische Physik, Freie Universität Berlin, Arnimallee 14, D-14195 Berlin, Germany \\ ${ }^{b}$ Institute of Nuclear Physics, Tashkent 100214, Uzbekistan
}

\begin{abstract}
We formulate the Collective Quantum Field Theory for three-dimensional bosonic optical lattices and evaluate its consequences in a mean-field approximation to two collective fields, proposed by Cooper et al. [19], and in a lowest-order Variational Perturbation Theory (VPT). It is shown that present mean-field approximation predicts some essential features of the experimentally observed dependence of the critical temperature on the coupling strength and a second - order quantum phase transition. In contrast to a recent prediction for atomic gases by Cooper et. al., we find no superfluid state with zero condensate fraction.

PACS numbers: $75.45+\mathrm{j}, 03.75 . \mathrm{Hh}, 75.30 . \mathrm{D}$
\end{abstract}

\section{INTRODUCTION}

Optical lattices are gases of ultracold atoms trapped in periodic potentials created by periodically arranged intersecting standing waves of laser light. The interest in experimental and theoretical investigations of these artificial crystals is caused by the two following factors [1]:

1) Neutral atoms in these optical lattices have several of attractive features that make them interesting candidates for the realization of a quantum computer [2].

2) They may be used to simulate various lattice models of fundamental importance in condensed matter physics. Since they permit studying in a controlled way solid-state physics, in which one can fine-tune the interaction strength for various geometries of the lattices. In particular, it is possible to control the Hamiltonian parameters and study various regimes of system parameter.

The lattice of bosons with short - range repulsive pair interaction trapped in an optical lattice may be described by a Hamiltonian of Bose-Hubbard type:

$$
H=-J \sum_{\langle\mathbf{i}, \mathbf{j}\rangle} \hat{b}_{\mathbf{i}}^{\dagger} \hat{b}_{\mathbf{j}}+\frac{U}{2} \sum_{\mathbf{i}}^{N_{s}} \hat{b}_{\mathbf{i}}^{\dagger} \hat{b}_{\mathbf{i}}^{\dagger} \hat{b}_{\mathbf{i}} \hat{b}_{\mathbf{i}}+\sum_{\mathbf{i}}^{N_{s}}\left(\varepsilon_{\mathbf{i}}-\mu\right) \hat{b}_{\mathbf{i}}^{\dagger} \hat{b}_{\mathbf{i}},
$$

where $\hat{b}_{\mathbf{i}}^{\dagger}$ and $\hat{b_{\mathbf{i}}}$ are the bosonic creation and annihilation operators on the site $i$; the sum over $\langle\mathbf{i}, \mathbf{j}\rangle$ includes only pairs of nearest neighbors; $J$ is the hopping amplitude, which is responsible for the tunneling of an atom from one site to another neighboring site; $U$ is the on site repulsion energy, and $N_{s}$ the number of sites.

At zero temperature with an integer filling factor $\nu \equiv N / N_{s}$, where $N$ is the total number of atoms, a system of bosons described by the Hamiltonian (11) could be on superfluid (SF) or in Mott insulator (MI) phase. Clearly the quantum phase transition (QPT) between these two phases is allocated by the dimensionless interaction strength parameter $u=U / J$. For small $u$, the hopping term dominates the system, so that it prefers to be in the SF phase. For large $u \gg 1$, on the other hands, the system exhibits a MI phase.

A critical interaction strength $u_{\text {crit }}=29.34$ was found for $d=3$ by Monte Carlo calculations [3] at a filling factor $\nu=1$, and agrees well with the experimental data [4].

To make for easier reading, we summarize some specific features of these two phases. The SF phase is characterized by a long-range correlation, a continuous (gapless) excitation spectrum and a finite compressibility. Since there exists a condensate with a finite number of particles, $n_{\mathbf{0}}$, the gauge symmetry is spontaneously broken in accordance with theorems by Bogoliubov and Ginibre. In contrast, in the MI phase, there is no long-range correlation or breaking of gauge symmetry. The excitation spectrum has a gap and the system is incompressible, since there is a fixed number of atoms per-site. The mobility of atoms is completely different in the two phases. In the SF phase they can easily move from one site to another site by tunneling, whereas in the MI phase, they are localized.

\footnotetext{
*Electronic address: h.k@fu-berlin.de

${ }^{\dagger}$ Electronic address: narzikulov@inp.uz

${ }^{\ddagger}$ Electronic address: rakhimovabd@yandex.ru
} 
Finite-temperature phases of optical lattices have been studied by quantum Monte Carlo (QMC) calculations as well as experimentally for $d=3$. As expected, the system behaves as a normal fluid (NF) at $T>T_{c}$. A most interesting observation was made in Refs. [3, 4]: In contrast to the system of dilute Bose gases, the critical temperature is downshifted at the transition to the MI phase.

Theoretical approaches based on the Bose-Hubbard model, which is not exactly soluble even in one dimension, have been summarized recently in textbooks [5-7]. Most of them use perturbative expansions in powers of $J / U$ and give qualitatively a good description of phase transition boundary [8, 9]. As to the nonperturbative approaches, they mainly exploit the Gutzwiller ansatz, where the wave function is expanded in local Fock states with variational coefficients. Although such an approach is good even in description of the dynamics of the system [10 13], since it is exact for $d \rightarrow \infty$, its reliability decreases dramatically for $d=1$.

Among various types of the existing mean - field theories in the literature the bosonic dynamical mean-field theory (B-DMFT) seems to be most powerful. Being originally proposed by Byczuk and Vollhardt [14] and further developed by Anders et al. [15] the B-DMFT maps the Bose-Hubbard model onto the self - consistent solution of a bosonic impurity model with coupling to a reservoir of normal and condensed bosons. The net output of this procedure is delightful. It gives as an accurate description of the phase diagrams, the condensate order parameter and other observables of the cubic lattice Bose-Hubbard model as it was obtained by QMC calculations. However, although the B-DMFT is numerically exact and flexible, it is computationally expensive, since one has to use continuous time QMC evaluations in order to solve its equations. Moreover, strictly speaking, the Hugenholtz - Pines theorem (see Subection IIB below) does not hold in B-DMFT (see Fig. 10 of Ref. [15]).

The application of non-perturbative renormalization group theory has revealed new scaling properties of optical lattices. Rancon and Dupuis [16] have recently shown that thermodynamic quantities of the Bose-Hubbard model can be expressed using universal scaling functions of the dilute Bose gas universality class.

As to the Bogoliubov theory, it provides an accurate description of the excitation spectrum for the SF phase, but fails to describe SF $\rightarrow$ MI transition. In fact, the first application of a mean-field approach was made in the Hartree-Fock-Popov (HFP) approximation to optical lattices by Stoof et. al. [17]. By studying the dependence of the condensate number $n_{\mathbf{0}}$ on $u$, i.e. $n_{\mathbf{0}}(U / J)$ they observed that $n_{\mathbf{0}}$ never reaches zero, even in the strong-coupling limit $(u \rightarrow \infty)$, implying that this approximation is unable to predict a QPT of SF $\rightarrow$ MI. In contrast to this, the two-loop approximation by the present authors in [18] suggests the existence of such a QPT, but the critical value of $u_{\text {crit }}$ was found to be rather small: $u_{\text {crit }}($ two-loop $) \approx 6$ for $d=3$. So, the question about the power of an approximation, based on mean-field theory, other than B-DMFT, to adequately describe phase diagrams of optical lattices remains still open. It is, therefore, desirable to develop a nonperturbative approach which would be suitable for dimensions $d=1,2,3$.

An alternative approach to the treatment of dilute Bose gases has recently been proposed by Fred Cooper et al. [19, 20] under the name of leading-order auxiliary field theory (LOAF). They found a way of fixing the degeneracy in the elimination of the interaction by auxiliary collective pair and density fields by choosing a special form of a generalized Hubbard-Stratonovitch transformation. Although their approach gives no QPT for a homogenous Bose gas at zero temperature, it predicts a desirable second order BEC transition at finite temperatures and exhibits a positive shift in the critical temperature $T_{c}$ that is consistent with Monte Carlo an other calculations [21, 22]. One of the novel features of that calculations is that for $T>T_{c}$ it predicts a novel type of superfluid phase that does not have a condensate [23]. Although such a phase has not been observed yet, it was justified by the existence of a nonzero anomalous density $\delta$, in the region $T_{c}<T \leq T^{*}$, where $T^{*}$ is the transition temperature to the normal phase.

In the present work we shall formulate a similar two-collective quantum field theory for discrete systems such as optical lattices and ask the following questions

- Does it predict a SF $\rightarrow$ MI quantum phase transition?

- Does it predict the suppression of $T_{c}$ at large $u$ ?

- Does it predict a new phase, mentioned above, for optical lattices either?

Our results will be compared with those of another well-known mean-field approximation, the Hartree - Fock Bogoliubov (HFB) approximation, which is widely used to describe BEC in homogeneous Bose gases and in triplons [24, 25] in magnetic insulators, and will also be extended here to optical lattices. Below we use $\hbar=k_{B}=1$.

The paper is organized as follows. In Sections II and III we shall derive Collective Quantum Field Theory and HFB approaches for optical lattices, respectively. The results and discussions will be presented in Section IV, and the conclusions will be stated in Section V. 


\section{COLLECTIVE QUANTUM FIELD THEORY OF 3D BOSE-HUBBARD MODEL}

In the Wannier representation the Euclidian action, corresponding to the Bose-Hubbard Hamiltonian is given by [18]

$$
\begin{aligned}
\mathcal{A}\left(\psi^{*}, \psi\right) & =\int_{0}^{\beta} d \tau\left\{\sum_{\mathbf{i}} \psi^{*}\left(\mathbf{x}_{\mathbf{i}}, \tau\right)\left[\partial_{\tau}-\mu\right] \psi\left(\mathbf{x}_{\mathbf{i}}, \tau\right)\right. \\
& -J \sum_{\langle i, j\rangle} \psi^{*}\left(\mathbf{x}_{\mathbf{i}}, \tau\right) \psi\left(\mathbf{x}_{j}, \tau\right) \\
& \left.+\frac{U}{2} \sum_{\mathbf{i}} \psi^{*}\left(\mathbf{x}_{\mathbf{i}}, \tau\right) \psi^{*}\left(\mathbf{x}_{\mathbf{i}}, \tau\right) \psi\left(\mathbf{x}_{\mathbf{i}}, \tau\right) \psi\left(\mathbf{x}_{\mathbf{i}}, \tau\right)\right\}
\end{aligned}
$$

where $\mu$ is the chemical potential and $\beta=1 / T$. The lattice points lie at the positions [26]

$$
\mathbf{x}_{\mathbf{i}}=\mathbf{i} a,
$$

where $a$ is the lattice spacing, and

$$
\mathbf{i} \equiv\left(i_{1}, i_{2}, \ldots, i_{d}\right)
$$

are integer-valued vectors.

The partition function $Z$, and the grand thermodynamic potential $\Omega$, can be found as:

$$
\begin{aligned}
Z & =\int D \psi^{*} D \psi e^{-\mathcal{A}\left(\psi^{*}, \psi\right)}, \\
\Omega & =-T \ln Z
\end{aligned}
$$

The ground state expectation value of an operator $\hat{O}\left(\psi^{*}, \psi\right)$ can be expressed as a functional integral:

$$
\langle\hat{O}\rangle=\frac{1}{Z} \int \mathcal{D} \psi^{*} \mathcal{D} \psi \hat{O}\left(\psi^{*}, \psi\right) e^{-\mathcal{A}\left(\psi^{*}, \psi\right)} .
$$

With the help of a Hubbard-Stratonovich transformation, the interaction term in (2) can be eliminated by adding to the action in the exponent of (5) a dummy action [27]:

$$
\mathcal{A}_{\text {pair }}\left[\psi^{*}, \psi, \Delta, \Delta^{*}\right]=\int_{0}^{\beta} d \tau \sum_{\mathbf{i}}\left\{\frac{1}{2 U}\left|\Delta\left(\mathbf{x}_{\mathbf{i}}, \tau\right)-U \psi\left(\mathbf{x}_{\mathbf{i}}, \tau\right) \psi\left(\mathbf{x}_{\mathbf{i}}, \tau\right)\right|^{2}\right\}
$$

containing a pair field $\Delta$. After this we form the path integral $\int \mathcal{D} \Delta \mathcal{D} \Delta^{*} e^{-\mathcal{A}_{\text {pair }}\left[\psi^{*}, \psi, \Delta, \Delta^{*}\right]}$, and integrate out the pair field. This produces a multiplication of the partition function $Z$ by a trivial constant factor.

It has been emphasized in [27] and the textbook [28] that this procedure is highly degenerate. Actually, instead of (8), one could just as well have introduced a plasmon field $\varphi(\mathbf{x}, \tau)$ by adding to the action in the exponent of (5) a dummy action

$$
\mathcal{A}_{\mathrm{pl}}\left[\psi^{*}, \psi, \varphi\right]=\int_{0}^{\beta} d \tau \sum_{\mathbf{i}}\left\{-\frac{1}{2 U}\left[\varphi\left(\mathbf{x}_{\mathbf{i}}, \tau\right)-U \psi^{*}\left(\mathbf{x}_{\mathbf{i}}, \tau\right) \psi\left(\mathbf{x}_{\mathbf{i}}, \tau\right)\right]^{2}\right\},
$$

and forming a functional integral integral $\int \mathcal{D} \varphi e^{-\mathcal{A}_{\text {pair }}\left[\psi^{*}, \psi, \varphi\right]}$, which again multiplies $Z$ by a trivial constant.

Diagrammatically, the degeneracy is caused by the fact that the sum of all collective field diagrams will always produce the same result if evaluated to all orders in perturbation theory. Each of these collective fields reproduces all effects of the interaction if it is integrated functionally. A difference appears, if the evaluation is restricted to a mean-field approximation. Then it depends on the dominance of certain dynamical effects which field is preferable.

In principle, we can also add a combination of $\mathcal{A}_{\text {pair }}$ and $\mathcal{A}_{\mathrm{pl}}$, and still leave the physical properties of the system unchanged. For instance $\mathcal{A}_{\mathrm{pl}} \cosh ^{2} \theta-\mathcal{A}_{\text {pair }} \sinh ^{2} \theta$. Diagrammatically, however, the degeneracy cannot be easily verified since a calculation of the diagrams to all order is really impossible. It can only be done to some finite order, for instance in a loop expansion, so that the mathematical equivalence is initially of little use.

One method to avoid the degeneracy and make the collective field approach unique has been pointed out a long time ago [29]. It is based on an extension of the standard effective action $\Gamma\left[\Psi^{*}, \Psi\right]$, whose functional expansion terms 
are the one-particle irreducible vertex functions of the theory. The symbol $\Psi$ denotes the expectations of the field $\psi(x, \tau)$. A unique version of collective fields can be introduced by going to a higher effective action $\mathcal{A}\left[\Psi^{*}, \Psi, \Delta, \Delta^{*}, \Phi\right]$. While the ordinary effective action $\Gamma\left[\Psi^{*}, \Psi\right]$ is derived from a Legendre transformation of the generating functional of the theory $W\left[\eta, \eta^{*}\right]$ in which additional source terms $\eta \psi^{*}+\eta^{*} \psi$ have been added to the action, the higher effective action is obtained from the Legendre transformation of a generating functional $W\left[\eta, \eta^{*}, j, K, K^{*}\right]$ in which additional sources have been added to the action coupled to the density and the pair fields. The higher effective action will depend on the expectations of the fields $\psi, \psi^{*}, \rho \propto \psi^{*} \psi, \Delta \propto \psi \psi$ and $\Delta^{*} \propto \psi^{*} \psi^{*}$. At the end, it must merely be extremized, and no extra functional integrals can cause any double-counting of Feynman diagrams. The expansion terms in the higher effective action are the two-particle irreducible vertex functions of the theory.

Another method that also abandons the fluctuations of the collective fields in favor of a collective classical field has been developed in recent years from a generalization of a variational approach to path integrals [30] to all orders in perturbation theory. It was extremely successful and has led to the most accurate theory of critical phenomena [31] so far, named Variational Perturbation Theory (VPT) (for a review paper see [32]).

A third method which has recently been proposed and applied [19, 20] uses the combination of both fully fluctuating collective fields implied by the above dummy action $\mathcal{A}_{\mathrm{pl}} \cosh ^{2} \theta-\mathcal{A}_{\text {pair }} \sinh ^{2} \theta$ for the particular value $\sinh \theta=1$. This choice is preferable if we want the mean-field approximation to exhibit excitations that have no energy gap, to comply with the Nambu-Goldstone theorem. After a trivial change of the normalization of plasmon and pair fields in the total action $\mathcal{A}+\mathcal{A}_{\mathrm{pl}} \cosh ^{2} \theta-\mathcal{A}_{\text {pair }} \sinh ^{2} \theta$ one arrives at

$$
\mathcal{A}=\mathcal{A}_{\psi}\left[\psi^{*}, \psi\right]+\mathcal{A}_{\varphi}[\varphi]+\mathcal{A}_{\Delta}\left[\Delta, \Delta^{*}\right]
$$

with

$$
\begin{aligned}
& \mathcal{A}_{\psi}\left[\psi^{*}, \psi\right]=\int_{0}^{\beta} d \tau \sum_{\mathbf{i}}\left\{\psi^{*}\left(\mathbf{x}_{\mathbf{i}}, \tau\right)\left[\partial_{\tau}-\mu+\varphi\left(\mathbf{x}_{\mathbf{i}}, \tau\right) \cosh \theta\right] \psi\left(\mathbf{x}_{\mathbf{i}}, \tau\right)\right. \\
& \left.-\frac{1}{2} \sinh \theta\left[\Delta \psi^{*}\left(\mathbf{x}_{\mathbf{i}}, \tau\right) \psi^{*}\left(\mathbf{x}_{\mathbf{i}}, \tau\right)+\Delta^{*} \psi\left(\mathbf{x}_{\mathbf{i}}, \tau\right) \psi\left(\mathbf{x}_{\mathbf{i}}, \tau\right)\right]\right\}-J \int_{0}^{\beta} d \tau \sum_{\mathbf{i}, \mathbf{j}} \psi^{*}\left(\mathbf{x}_{\mathbf{i}}, \tau\right) \psi\left(\mathbf{x}_{\mathbf{j}}, \tau\right), \\
& \mathcal{A}_{\varphi}[\varphi]=-\int_{0}^{\beta} d \tau \sum_{\mathbf{i}} \frac{\varphi^{2}\left(\mathbf{x}_{\mathbf{i}}, \tau\right)}{2 U}, \quad \mathcal{A}_{\Delta}\left[\Delta, \Delta^{*}\right]=\int_{0}^{\beta} d \tau \sum_{\mathbf{i}} \frac{\Delta\left(\mathbf{x}_{\mathbf{i}}, \tau\right) \Delta^{*}\left(\mathbf{x}_{\mathbf{i}}, \tau\right)}{2 U} .
\end{aligned}
$$

At the level of for fully fluctuating fields $\varphi, \Delta, \Delta^{*}$, the parameter $\theta$ is still arbitrary, which will be fixed in the next section.

Now we consider separately two regions, with and without a condensed phase.

\section{A. Condensed phase}

In this phase, the $U(1)$ gauge symmetry is spontaneously broken. It can be studied after a Bogoliubov shift of the field 33 ]

with

$$
\psi\left(x_{\mathbf{i}}, \tau\right)=\psi_{\mathbf{0}}+\widetilde{\psi}\left(x_{\mathbf{i}}, \tau\right)
$$

$$
\psi_{\mathbf{0}}=\sqrt{\nu n_{\mathbf{0}}}
$$

where the $n_{\mathbf{0}}=N_{\mathbf{0}} / N$ is the condensate fraction. It is a constant in the absence of a magnetic trap. The fluctuating field $\tilde{\psi}(x, \tau)$ must satisfy the condition:

$$
\int_{0}^{\beta} d \tau \sum_{\mathbf{i}} \tilde{\psi}\left(x_{\mathbf{i}}, \tau\right)=0 .
$$

Substituting (13) into (11), and decomposing the quantum field $\tilde{\psi}\left(\mathbf{x}_{\mathbf{i}}, t\right)$ into its real and imaginary parts $\psi_{1}\left(\mathbf{x}_{\mathbf{i}}, t\right)$ and $\psi_{2}\left(\mathbf{x}_{\mathbf{i}}, t\right)$ as

$$
\begin{aligned}
\tilde{\psi}\left(\mathbf{x}_{\mathbf{i}}, t\right) & =\frac{1}{\sqrt{2}}\left(\psi_{1}\left(\mathbf{x}_{\mathbf{i}}, t\right)+i \psi_{2}\left(\mathbf{x}_{\mathbf{i}}, t\right)\right), \\
\tilde{\psi}^{*}\left(\mathbf{x}_{\mathbf{i}}, t\right) & =\frac{1}{\sqrt{2}}\left(\psi_{1}\left(\mathbf{x}_{\mathbf{i}}, t\right)-i \psi_{2}\left(\mathbf{x}_{\mathbf{i}}, t\right)\right),
\end{aligned}
$$


we may separate the action as follows:

$$
\mathcal{A}=\mathcal{A}_{0}+\mathcal{A}_{2}+\mathcal{A}_{\Delta}+\mathcal{A}_{\varphi}
$$

with

$$
\begin{aligned}
\mathcal{A}_{0}= & -N_{s} \beta \nu n_{\mathbf{0}}\left(\mu+J z_{0}\right)+\nu n_{\mathbf{0}} \sum_{\mathbf{i}} \int_{0}^{\beta} d \tau\left[\cosh \theta \varphi\left(x_{\mathbf{i}}, \tau\right)\right. \\
& \left.-\frac{1}{2} \sinh \theta\left(\Delta\left(x_{\mathbf{i}}, \tau\right)+\Delta^{*}\left(x_{\mathbf{i}}, \tau\right)\right)\right], \\
\mathcal{A}_{2}= & \frac{1}{2} \sum_{\mathbf{i}} \int_{0}^{\beta} d \tau \sum_{a, b=1,2}\left[i \varepsilon_{a b} \tilde{\psi}_{a}\left(x_{\mathbf{i}}, \tau\right) \partial_{\tau} \tilde{\psi}_{b}\left(x_{\mathbf{i}}, \tau\right)\right. \\
& \left.+\tilde{\psi}_{a}\left(x_{\mathbf{i}}, \tau\right) X_{a} \tilde{\psi}_{b}\left(x_{\mathbf{i}}, \tau\right) \delta_{a b}\right] \\
& -\frac{J}{2} \int_{0}^{\beta} d \tau \sum_{\langle\mathbf{i}, \mathbf{j}\rangle} \sum_{a} \tilde{\psi}_{a}\left(x_{\mathbf{i}}, \tau\right) \tilde{\psi}_{a}\left(x_{j}, \tau\right),
\end{aligned}
$$

where $\mathcal{A}_{\Delta}$ and $\mathcal{A}_{\varphi}$ are given in (12), $\varepsilon_{a b}$ is an antisymmetric tensor with $\varepsilon_{12}=-\varepsilon_{21}=1, z_{0}=2 d$, and

$$
\begin{aligned}
& X_{1}=-\mu+\varphi\left(x_{\mathbf{i}}, \tau\right) \cosh \theta-\frac{1}{2} \sinh \theta\left(\Delta^{*}\left(x_{\mathbf{i}}, \tau\right)+\Delta\left(x_{\mathbf{i}}, \tau\right)\right), \\
& X_{2}=-\mu+\varphi\left(x_{\mathbf{i}}, \tau\right) \cosh \theta+\frac{1}{2} \sinh \theta\left(\Delta^{*}\left(x_{\mathbf{i}}, \tau\right)+\Delta\left(x_{\mathbf{i}}, \tau\right)\right) .
\end{aligned}
$$

For a homogenous, system the condensate is uniform and it is convenient to decompose the fluctuations into a Fourier series as [34, 35]

$$
\tilde{\psi}_{a}\left(\mathbf{x}_{\mathbf{i}}, \tau\right)=\frac{1}{\beta \sqrt{N_{s}^{d}}} \sum_{\mathbf{q}, \omega_{n}}^{\prime} \int_{0}^{\beta} \psi_{a}\left(\mathbf{q}, \omega_{n}\right) e^{-i \omega_{n} \tau} \exp \left[i \mathbf{x}_{\mathbf{i}} \mathbf{p}_{\mathbf{q}}\right]
$$

where $\omega_{n}=2 \pi n T$ are Matsubara frequencies, and $\mathbf{p}_{\mathbf{q}} \equiv\left\{q_{1}, q_{2}, \ldots, q_{d}\right\} 2 \pi / N_{s} a$, with $q_{\mathbf{i}}$ running from 1 to $N_{s}-1$ are the discrete-valued momentum vectors in the Brillouin zone. The momentum sum is explicitly

$$
\frac{1}{N_{s}} \sum_{\mathbf{q}}^{\prime} \equiv \frac{1}{N_{s}^{d}} \sum_{q_{1}=1}^{N_{s}-1} \sum_{q_{2}=1}^{N_{s}-1} \cdots \sum_{q_{d}=1}^{N_{s}-1}
$$

The prime on the symbol indicates that the $\mathbf{p}=0$-mode is omitted since it is contained in the subtracted $\psi_{\mathbf{0}}$. This will be useful to avoid possible infrared divergencies, especially for $d<3$.

In momentum space, the quadratic term $\mathcal{A}_{2}$ reads

$$
\mathcal{A}_{2}=\frac{1}{2} \sum_{\mathbf{q}, \mathbf{q}^{\prime}, m, n} \psi_{a}\left(\mathbf{q}, \omega_{n}\right) G_{a b}^{-1}\left(\mathbf{q}, \omega_{n} ; \mathbf{q}^{\prime}, \omega_{m}\right) \psi_{b}\left(\mathbf{q}^{\prime}, \omega_{m}\right)
$$

with the propagator

$$
G\left(\omega_{n}, \mathbf{q}\right)=\frac{1}{\omega_{n}^{2}+\mathcal{E}^{2}(\mathbf{q})}\left(\begin{array}{cc}
\varepsilon(\mathbf{q})+X_{2}-J z_{0} & \omega_{n} \\
-\omega_{n} & \varepsilon(\mathbf{q})+X_{1}-J z_{0}
\end{array}\right)
$$

where the bare dispersion $\varepsilon(\mathbf{q})$ and phonon dispersion $\mathcal{E}(\mathbf{q})$ are given by

$$
\begin{aligned}
& \varepsilon(\mathbf{q})=2 J\left(d-\sum_{\alpha=1}^{d} \cos \left(2 \pi q_{\alpha} / N_{s}\right)\right) \\
& \mathcal{E}(\mathbf{q})=\sqrt{\left(X_{1}+\varepsilon(\mathbf{q})-J z_{0}\right)\left(X_{2}+\varepsilon(\mathbf{q})-J z_{0}\right)} .
\end{aligned}
$$

In the long-wavelength limit, $\varepsilon(\mathbf{q})$ behaves like

$$
\varepsilon(\mathbf{q}) \approx J \frac{4 \pi^{2}}{N_{s}^{2}} \mathbf{q}^{2}=J a^{2} \mathbf{p}^{2}+\ldots
$$


By comparison with the usual momentum-dependence of a free single-particle energy $\mathbf{p}^{2} / 2 M$ we identify the particle mass $M=1 / 2 J a^{2}$.

Note that in coordinate space the Green function is defined by

$$
\begin{aligned}
G_{a b}\left(\mathbf{x}_{\mathbf{i}}, \tau ; \mathbf{x}_{\mathbf{j}}, \tau^{\prime}\right) \equiv & G_{a b}\left(\mathbf{x}_{\mathbf{i}}-\mathbf{x}_{\mathbf{j}}, \tau-\tau^{\prime}\right) \\
= & \left\langle\psi_{a}\left(\mathbf{x}_{\mathbf{i}}, \tau\right) \psi_{b}\left(\mathbf{x}_{\mathbf{j}}, \tau^{\prime}\right)\right\rangle \\
= & \frac{1}{N_{s} \beta} \sum_{n} \sum_{q} e^{i \omega_{n}\left(\tau-\tau^{\prime}\right)} e^{i \mathbf{q}\left(\mathbf{x}_{\mathbf{i}}-\mathbf{x}_{\mathbf{j}}\right)} \\
& \times G_{a b}\left(\omega_{n}, \mathbf{q}\right) .
\end{aligned}
$$

The thermodynamics of the system can be calculated from the partition function $Z$ functional integral over all fields $\psi_{1}, \psi_{2}, \varphi, \Delta$ and $\Delta^{*}$ fields

$$
Z=\int \mathcal{D} \psi_{1} \mathcal{D} \psi_{2} \mathcal{D} \varphi \mathcal{D} \Delta \mathcal{D} \Delta^{*} e^{-\mathcal{A}_{0}-\mathcal{A}_{2}-\mathcal{A}_{\Delta}-\mathcal{A}_{\varphi}} .
$$

The first integrations by $\psi_{1}$ and $\psi_{2}$ are Gaussian and may be evaluated easily by using well-known formula

$$
\begin{aligned}
& \int \mathcal{D} \psi_{1} \mathcal{D} \psi_{2} \exp \left[-\frac{1}{2} \sum_{a, b=1,2} \int \psi_{a}(x) G_{a b}^{-1}(x, y) \psi_{b}(y) d x d y\right. \\
& \left.-\int j_{1}(x) \psi_{1}(x) d x-\int j_{2}(x) \psi_{2}(x) d x\right] \\
= & \sqrt{\operatorname{Det} G} \exp \left[\sum_{a, b=1,2} \int j_{a}(x) G_{a b}(x, y) j_{b}(y) d x d y\right] .
\end{aligned}
$$

The integrations over the fluctuating collective fields, however, cannot be performed exactly, since they are nontrivially contained in $\sqrt{\operatorname{Det} G}$. As usual in these circumstances, we resort to the saddle-point approximation [27, 36]. In the absence of a trap, we may assume the saddle point to lie at constant values of $\varphi\left(x_{\mathbf{i}}, \tau\right)$ and $\Delta\left(x_{\mathbf{i}}, \tau\right)$ :

$$
\begin{aligned}
& \varphi\left(x_{\mathbf{i}}, \tau\right)=\varphi_{0}, \\
& \Delta\left(x_{\mathbf{i}}, \tau\right)=\Delta^{*}\left(x_{\mathbf{i}}, \tau\right)=\Delta_{0} .
\end{aligned}
$$

Then the integrals over $\psi_{a}$ become trivial and we may use the formula $\operatorname{Det} G=e^{\operatorname{Tr} \ln G}$ in Eqs. (29) and (30) to derive the following effective potential:

$$
\begin{aligned}
\Omega= & \frac{T}{2} \sum_{q} \sum_{n} \ln \left(\omega_{n}^{2}+\mathcal{E}^{2}(\mathbf{q})\right)+N_{s} \nu n_{\mathbf{0}}\left(\varphi^{\prime}-\Delta\right) \\
& +\frac{N_{s} \Delta^{2}}{2 U \sinh ^{2} \theta}-\frac{N_{s}\left(\varphi^{\prime}+\mu+J z_{0}\right)^{2}}{2 U \cosh ^{2} \theta}
\end{aligned}
$$

with

$$
\Delta \equiv \Delta_{0} \sinh \theta, \quad \varphi^{\prime}=\varphi_{0} \cosh \theta-\mu-J z_{0} .
$$

The spectrum of density fluctuations is now from (26):

$$
\mathcal{E}^{2}(\mathbf{q})=\left(\varepsilon(\mathbf{q})+\varphi^{\prime}-\Delta\right)\left(\varepsilon(\mathbf{q})+\varphi^{\prime}+\Delta\right) .
$$

The sum over $\mathbf{p}$ may be calculated in $d=3$ by approximating (22) as follows

$$
\frac{1}{N_{s}} \sum_{\mathbf{q}} f(\varepsilon(\mathbf{q})) \rightarrow \int_{0}^{1} d q_{1} d q_{2} d q_{3} f\left(\varepsilon_{\mathbf{q}}\right),
$$

with the lattice dispersion:

$$
\varepsilon_{\mathbf{q}}=2 J \sum_{\alpha=1}^{3}\left[1-\cos \pi q_{\alpha}\right]
$$

Note that on lattices, the momentum integrals are always finite so that there is no need for renormalizing the coupling constant. This is in contrast to atomic gases. However, if we want to express the coupling constant in terms of the scattering length $a_{s}$ that is observable at low-energy atomic gases, where the quadratic coupling constant $g$ must be renormalized to a finite value $g_{R}$ by the addition of a diverging integral $1 / g_{R}=1 / g+\int d^{3} p /(2 \pi)^{3} \varepsilon(\mathbf{p})$, the 
relation $a_{s}=M g_{R} / 4 \pi$ can only be employed only after a corresponding addition of a finite sum [see the remarks after Eq. (93)].

Another remark concerns the frequency sum in (32), which is initially divergent. In fact, to evaluate a frequency sum such as $\sum_{n=-\infty}^{\infty} \ln \left(a^{2}+\omega_{n}^{2}\right)$ with $\omega_{n}=2 \pi n T$, one must first differentiate it with respect to $a$, perform the summation over $n$, and integrate the result over $a$ [37]. This procedure gives an additional divergent constant, which may be removed by an additive renormalization of the energy [38]. The subtraction can actually be justified by calculating the path integral as a product of individual integrals for each slice of a sliced time axis, as introduced originally by Feynman [37].

Therefore, in the thermodynamic potential $\Omega$, one subtracts from $\Omega$ the one for the "ideal" case

$$
\begin{aligned}
\Omega(U=T=0) & =\frac{1}{2} \sum_{q}\left(\varepsilon(\mathbf{q})-\mu-J z_{0}\right) \\
& =\frac{1}{2} \sum_{q}\left(\varepsilon(\mathbf{q})+\varphi^{\prime}\right)
\end{aligned}
$$

and deals only with the subtracted expression

$$
\begin{aligned}
\Omega_{\mathrm{ren}}= & \Omega(U, T)-\Omega(U=0, T=0) \\
= & \frac{1}{2} \sum_{q}\left(\mathcal{E}(\mathbf{q})-\varepsilon(\mathbf{q})-\varphi^{\prime}\right)+N_{s} \nu n_{\mathbf{0}}\left(\varphi^{\prime}-\Delta\right) \\
& +\frac{N_{s} \Delta^{2}}{2 U \sinh ^{2} \theta}-\frac{N_{s}\left(\varphi^{\prime}+\mu+J z_{0}\right)^{2}}{2 U \cosh ^{2} \theta} \\
& +T \sum_{q} \ln \left(1-e^{-\beta \mathcal{E}(\mathbf{q})}\right),
\end{aligned}
$$

where we have performed summation by Matsubara frequency by using formula

$$
\sum_{n=-1}^{\infty} \ln \left(\omega_{n}^{2}+a^{2}\right)=a \beta+2 \ln \left(1-e^{-\beta a}\right)+\text { divergent const. }
$$

For brevity, we shall suppress writing down the subtraction in $\Omega_{\text {ren }}$.

In equilibrium, the thermodynamic potential reaches a minimum with respect to parameters $n_{\mathbf{0}}, \varphi^{\prime}$ and $\Delta$. Thus we minimize $\Omega$ with respect to $n_{\mathbf{0}}$

$$
\frac{\partial \Omega}{\partial n_{\mathbf{0}}}=N_{s} \nu\left(\varphi^{\prime}-\Delta\right)=0
$$

and get

$$
\varphi^{\prime}=\Delta
$$

Inserting this into (34) leads to the well-known Bogoliubov phonon dispersion

$$
\mathcal{E}(\mathbf{q})=\sqrt{\varepsilon(\mathbf{q})} \sqrt{\varepsilon(\mathbf{q})+2 \Delta},
$$

which is linear in $\mathbf{q}$ for small momentum, thus respecting the Nambu-Goldstone theorem.

Minimizing thermodynamic potential $\Omega$ with respect to $\Delta$ gives the equation:

$$
\Delta=U \sinh ^{2} \theta\left[\nu n_{\mathbf{0}}+\frac{\Delta}{N_{s}} \sum_{\mathbf{q}} \frac{c_{\mathbf{q}}}{\mathcal{E}(\mathbf{q})}\right]
$$

where $c_{\mathbf{q}}$ stands for

$$
\begin{aligned}
& c_{\mathbf{q}}=\frac{1}{2}+f_{\beta}(\mathcal{E}(\mathbf{q}))=\frac{1}{2} \operatorname{coth}(\beta \mathcal{E}(\mathbf{q}) / 2), \\
& f_{\beta}(\omega)=1 /\left(e^{\beta \omega}-1\right) .
\end{aligned}
$$

Minimizing $\Omega$ with respect to $\varphi^{\prime}$, thereby taking into account the relation $\partial \mathcal{E}(\mathbf{q}) / \partial \varphi^{\prime}=\left(\varepsilon(\mathbf{q})+\varphi^{\prime}\right) / \mathcal{E}(\mathbf{q})$, gives the following equation:

$$
N_{s} \nu n_{\mathbf{0}}+\sum_{q}\left[\frac{\left(\varepsilon(\mathbf{q})+\varphi^{\prime}\right) c_{\mathbf{q}}}{\mathcal{E}(\mathbf{q})}-\frac{1}{2}\right]-\frac{N_{s}\left(\varphi^{\prime}+\mu+J z_{0}\right)}{U \cosh ^{2} \theta}=0 .
$$

This will serve to determine of uncondensed fraction $n_{\mathbf{u}}$. 


\section{B. Normal and anomalous densities}

According to the general rules of statistical mechanics, the total number of particles $N$ is conjugate to the chemical potential:

Applying this to (38) gives

$$
N=-\left(\frac{\partial \Omega}{\partial \mu}\right)_{T, V}
$$

$$
N=\frac{N_{s}\left(\varphi^{\prime}+\mu+J z_{0}\right)}{U \cosh ^{2} \theta}
$$

Using (47) in (45), we obtain

$$
N=N_{s} \nu n_{\mathbf{0}}+\sum_{q}\left[\frac{\left(\varepsilon(\mathbf{q})+\varphi^{\prime}\right) c_{\mathbf{q}}}{\mathcal{E}(\mathbf{q})}-\frac{1}{2}\right] \equiv N_{\mathbf{0}}+N_{\mathbf{u}}
$$

Here $N_{\mathbf{0}}$ is a total number of condensed atoms, and $n_{\mathbf{0}}=N_{\mathbf{0}} / N_{s} \nu$ is the condensate fraction. The uncondensed atoms have a fraction

$$
n_{\mathbf{u}}=\frac{N_{\mathbf{u}}}{N}=\frac{1}{\nu N_{s}} \sum_{q}\left[\frac{\left(\varepsilon(\mathbf{q})+\varphi^{\prime}\right) c_{\mathbf{q}}}{\mathcal{E}(\mathbf{q})}-\frac{1}{2}\right]
$$

It satisfies the trivial relation $n_{\mathbf{0}}+n_{\mathbf{u}}=1$.

Note that, the term $-\frac{1}{2}$ in the square bracket of (49) is due to the renormalization procedure (38), and guarantees that at $T=0$ all particles of the ideal gas (which has $U=0$ and $\Delta=0$ ) are condensed, so that $n_{\mathbf{u}}(U=0, T=0)=0$.

When the $\mathrm{U}(1)$ gauge symmetry is broken, a Bose system is characterized not only by the expectation values of the fluctuating part of the $\psi$-field with the normal density $n_{\mathbf{u}}=\left\langle\tilde{\psi}^{*} \tilde{\psi}\right\rangle$, but also with anomalous density, defined by

$$
\delta\left(x_{\mathbf{i}}, \tau, x_{j}, \tau^{\prime}\right)=\left\langle\tilde{\psi}\left(x_{\mathbf{i}}, \tau\right) \tilde{\psi}\left(x_{j}, \tau^{\prime}\right)\right\rangle .
$$

Clearly, for homogenous system in the equilibrium, in particular, for periodic optical lattices without magnetic trap, $\delta$ does not depend on coordinates, i.e. $\delta\left(x_{\mathbf{i}}, \tau, x_{j}, \tau^{\prime}\right)=$ const as was emphasized in [39]. Omission of the anomalous averages makes all calculations not self-consistent, the dynamics non-conserving, the thermodynamics incorrect. It ruins the order of the phase transition and renders the system unstable. It was also shown in [39] that a $\delta=0$ type of mean-field approach referred in the literatures as Hartree-Fock-Popov (HFP) approximations [24] leads to a discontinuity in the magnetization curve of antiferromagnetic material with the triplon BEC. Thus we must always allow for $\delta \neq 0$.

Let us calculate this expectation value from the formula

$$
\begin{aligned}
\delta & =\frac{1}{\nu}\left\langle\tilde{\psi}\left(x_{\mathbf{i}}, \tau\right) \tilde{\psi}\left(x_{\mathbf{i}}, \tau\right)\right\rangle \\
& =\frac{1}{2 \nu}\left[\left\langle\tilde{\psi}_{1}\left(x_{\mathbf{i}}, \tau\right) \tilde{\psi}_{1}\left(x_{\mathbf{i}}, \tau\right)\right\rangle-\left\langle\tilde{\psi}_{2}\left(x_{\mathbf{i}}, \tau\right) \tilde{\psi}_{2}\left(x_{\mathbf{i}}, \tau\right)\right\rangle\right] \\
& =\frac{1}{2 \nu}\left[G_{11}(0)-G_{22}(0)\right] .
\end{aligned}
$$

In momentum space, the propagator can be rewritten as

$$
G_{a b}\left(\omega_{n}, \mathbf{q}\right)=\frac{1}{\omega_{n}^{2}+\mathcal{E}^{2}(\mathbf{q})}\left(\begin{array}{lr}
\varepsilon(\mathbf{q})+2 \Delta & \omega_{n} \\
-\omega_{n} & \varepsilon(\mathbf{q})
\end{array}\right),
$$

where we used equations (20), (24), and (42). Using in (51) the equations (28) and (52), one obtains

$$
\begin{aligned}
\delta & =\frac{1}{2 \nu N_{s} \beta} \sum_{n} \sum_{\mathbf{q}} \frac{2 \Delta}{\omega_{n}^{2}+\mathcal{E}^{2}(\mathbf{q})} \\
& =\frac{\Delta}{\nu N_{s}} \sum_{\mathbf{q}} \frac{c_{\mathbf{q}}}{\mathcal{E}(\mathbf{q})}=\frac{\Delta}{\nu N_{s}} \sum_{\mathbf{q}} \frac{1}{\mathcal{E}(\mathbf{q})}\left(\frac{1}{2}+\frac{1}{e^{\beta \mathcal{E}(\mathbf{q})}-1}\right) .
\end{aligned}
$$

In terms of $\delta$, the $\Delta$-equation (43) may be rewritten in the following compact form

$$
\Delta=U \nu\left(n_{\mathbf{0}}+\delta\right) \sinh ^{2} \theta,
$$

with $n_{\mathbf{0}}=1-n_{\mathbf{u}}$, and $n_{\mathbf{u}}$ given by (49). 
It is well known that the Goldstone theorem for a dilute Bose gas with a spontaneous broken symmetry is equivalent to the celebrated Hugenholtz-Pines theorem [40], according to which self-energy $\Sigma_{\mathrm{cl}}$ and the anomalous self-energy $\Delta_{\mathrm{cl}}$ satisfy

$$
\Sigma_{\mathrm{cl}}-\Delta_{\mathrm{cl}}=\mu .
$$

In the Appendix A we shall show that a similar equation holds for optical lattices:

$$
\Sigma_{\mathrm{cl}}-\Delta_{\mathrm{cl}}=\mu+J z_{0},
$$

with $\Sigma_{\mathrm{cl}}=\varphi_{0} \cosh \theta, \Delta_{\mathrm{cl}}=\Delta$.

The only parameter, that so far remains free in the initial action (11), is $\theta$. It may be chosen such that the quasiparticle energy $\mathcal{E}(\mathbf{q})$ reduces, in the one-loop approximation [18], to the gapless Bogoliubov dispersion

$$
\mathcal{E}(\mathbf{q})_{\text {oneloop }}=\sqrt{\varepsilon(\mathbf{q})} \sqrt{\varepsilon(\mathbf{q})+2 U \nu} .
$$

Indeed, in this approximation we get from (54) $\Delta \approx U \nu \sinh ^{2} \theta$, and from (42) $\mathcal{E}(\mathbf{q}) \approx \sqrt{\varepsilon(\mathbf{q})} \sqrt{\varepsilon(\mathbf{q})+2 U \nu \sinh ^{2} \theta}$. This is the place where we fix the $\theta$ to satisfy

$$
\sinh ^{2} \theta=1, \quad \cosh ^{2} \theta=2,
$$

as was announced earlier.

Summarizing this section, we present the full expression for $\Omega$ :

$$
\begin{aligned}
\Omega= & \frac{1}{2} \sum_{\mathbf{q}}[\mathcal{E}(\mathbf{q})-\varepsilon(\mathbf{q})-\Delta] \\
& +\frac{N_{s} \Delta^{2}}{2 U}-\frac{N_{s}\left(\Delta+\mu+J z_{0}\right)^{2}}{4 U} \\
& +T \sum_{\mathbf{q}} \ln \left(1-e^{-\beta \mathcal{E}(\mathbf{q})}\right),
\end{aligned}
$$

with

$$
\mu=2 \nu U-\Delta-J z_{0} .
$$

The last equation follows from (47). The self energy $\Delta$ in (59) and (60) is defined through the following set of nonlinear algebraic equations:

$$
\begin{aligned}
& \Delta=U \nu\left(n_{0}+\delta\right), \quad n_{0}=1-n_{\mathbf{u}}, \\
& n_{\mathbf{u}}=\frac{1}{\nu N_{s}} \sum_{\mathbf{q}}\left[\frac{c_{\mathbf{q}}(\varepsilon(\mathbf{q})+\Delta)}{\mathcal{E}(\mathbf{q})}-\frac{1}{2}\right], \\
& \delta=\frac{\Delta}{\nu N_{s}} \sum_{\mathbf{q}} \frac{c_{\mathbf{q}}}{\mathcal{E}(\mathbf{q})},
\end{aligned}
$$

where $c_{\mathbf{q}}$ is given in (44) and $U, J, \nu, T$ are input parameters.

\section{Symmetric phase}

When $n_{\mathbf{0}}=0$, the Hamiltonian (10) is symmetric under the transformation $\psi \rightarrow e^{i \alpha} \psi$ and equation (40) makes no sense. Then $\varphi^{\prime} \neq \Delta$, and the energy spectrum has a gap with the dispersion

$$
\mathcal{E}(\mathbf{q})=\sqrt{\left(\varepsilon(\mathbf{q})+\varphi^{\prime}-\Delta\right)\left(\varepsilon(\mathbf{q})+\varphi^{\prime}+\Delta\right)} .
$$

The main equations in this regime with $T>T_{c}$ are

$$
\begin{aligned}
& \Delta=U \nu \delta, \quad \delta=\frac{\Delta}{\nu N_{s}} \sum_{\mathbf{q}} \frac{c_{\mathbf{q}}}{\mathcal{E}(\mathbf{q})}, \\
& \nu=\frac{1}{N_{s}} \sum_{\mathbf{q}}\left[\frac{\left(\varepsilon(\mathbf{q})+\varphi^{\prime}\right) c_{\mathbf{q}}}{\mathcal{E}(\mathbf{q})}-\frac{1}{2}\right] .
\end{aligned}
$$


The set of equations (63) with the energy spectrum (62) may have a solution $\Delta \neq 0, \varphi^{\prime}>\Delta$, leading to an exotic state with no condensate but with a finite anomalous density: $n_{\mathbf{0}}=0, \delta \neq 0$. It was shown in Ref. [23] that this phase has a nonzero SF fraction. The upper boundary of such a state was denoted by $T^{*}$, and was determined by solving the equations (63) with $\Delta=0, \varphi^{\prime}>0$. Thus it was theoretically predicted that ultracold dilute atomic gases posses a superfluid state at $T_{c}<T \leq T^{*}$ without Bose condensation in the one-body channel 23]. However, up to date, such states have not been observed experimentally. In Sect. IV we shall investigate the possible existence of such a state for optical lattices, with a negative outcome.

\section{VARIATIONAL PERTURBATION THEORY IN OPTICAL LATTICES}

It is interesting to compare our result with those of Variational Perturbation Theory [31]. To lowest order, this is equivalent to the HFB approximation used in the operator formalism [41]. To do this, let us formulate the HFB approximation for optical lattices in the functional integral framework.

Starting point is again (2) in which we perform the Bogoliubov shift (13) and separate the action as follows

$$
\mathcal{A}=\mathcal{A}_{(0)}+\mathcal{A}_{(1)}+\mathcal{A}_{(2)}+\mathcal{A}_{(3)}+\mathcal{A}_{(4)},
$$

where

$$
\begin{aligned}
\mathcal{A}_{(0)}= & \beta N_{s} \nu n_{\mathbf{0}}\left[\frac{U}{2} \nu n_{\mathbf{0}}-\mu-J z_{0}\right], \\
\mathcal{A}_{(1)}= & \sqrt{\nu n_{\mathbf{0}}}\left[-\mu-J z_{0}+U \nu n_{\mathbf{0}}\right] \int d \tau \sum_{\mathbf{i}}\left(\tilde{\psi}\left(\mathbf{x}_{\mathbf{i}}, \tau\right)+\tilde{\psi}^{*}\left(\mathbf{x}_{\mathbf{i}}, \tau\right)\right), \\
\mathcal{A}_{(2)}= & \int_{0}^{\beta} d \tau\left\{\sum_{\mathbf{i}} \tilde{\psi}^{*}\left(\mathbf{x}_{\mathbf{i}}, \tau\right)\left[\partial_{\tau}-\mu\right] \tilde{\psi}\left(\mathbf{x}_{\mathbf{i}}, \tau\right)+\frac{U}{2} \nu n_{\mathbf{0}}\right. \\
& \left.\times \sum_{\mathbf{i}}\left[\tilde{\psi}^{2}\left(\mathbf{x}_{\mathbf{i}}, \tau\right)+4 \tilde{\psi}^{*}\left(\mathbf{x}_{\mathbf{i}}, \tau\right) \tilde{\psi}\left(\mathbf{x}_{\mathbf{i}}, \tau\right)+\tilde{\psi}^{*}\left(\mathbf{x}_{\mathbf{i}}, \tau\right) \tilde{\psi}^{*}\left(\mathbf{x}_{\mathbf{i}}, \tau\right)\right]-J \sum_{\langle i, j\rangle} \tilde{\psi}^{*}\left(\mathbf{x}_{\mathbf{i}}, \tau\right) \tilde{\psi}\left(\mathbf{x}_{j}, \tau\right)\right\}, \\
\mathcal{A}_{(3)}= & U \sqrt{\nu n_{\mathbf{0}}} \int_{0}^{\beta} d \tau \sum_{\mathbf{i}}\left[\tilde{\psi}^{*}\left(\mathbf{x}_{\mathbf{i}}, \tau\right) \tilde{\psi}^{2}\left(\mathbf{x}_{\mathbf{i}}, \tau\right)+\tilde{\psi}^{*}\left(\mathbf{x}_{\mathbf{i}}, \tau\right) \tilde{\psi}^{*}\left(\mathbf{x}_{\mathbf{i}}, \tau\right) \tilde{\psi}\left(\mathbf{x}_{\mathbf{i}}, \tau\right)\right], \\
\mathcal{A}_{(4)}= & \frac{U}{2} \int_{0}^{\beta} d \tau \sum_{\mathbf{i}}\left[\tilde{\psi}^{*}\left(\mathbf{x}_{\mathbf{i}}, \tau\right) \tilde{\psi}\left(\mathbf{x}_{\mathbf{i}}, \tau\right)\right]^{2} .
\end{aligned}
$$

After this we add and subtract following terms

$$
\mathcal{A}_{(\Sigma)}=\int_{0}^{\beta} d \tau \sum_{\mathbf{i}}\left\{\Sigma_{\mathrm{cl}} \tilde{\psi}^{*}\left(\mathbf{x}_{\mathbf{i}}, \tau\right) \tilde{\psi}\left(\mathbf{x}_{\mathbf{i}}, \tau\right)+\frac{1}{2} \Delta_{\mathrm{cl}}\left[\tilde{\psi}^{*}\left(\mathbf{x}_{\mathbf{i}}, \tau\right) \tilde{\psi}^{*}\left(\mathbf{x}_{\mathbf{i}}, \tau\right)+\tilde{\psi}\left(\mathbf{x}_{\mathbf{i}}, \tau\right) \tilde{\psi}\left(\mathbf{x}_{\mathbf{i}}, \tau\right)\right]\right\},
$$

with variational parameters $\Sigma_{\mathrm{cl}}$ and $\Delta_{\mathrm{cl}}$. The subscripts cl emphasize that these are variational parameters which, in contrast to the earlier fields $\varphi$ and $\Delta$, are not meant to be functionally integrated.

Using again real and imaginary parts of the complex fields $\tilde{\psi}, \tilde{\psi}^{*}$ as in (16), we rewrite $\mathcal{A}$ as

$$
\mathcal{A}=\mathcal{A}_{(0)}+\mathcal{A}_{\text {free }}+\mathcal{A}_{\text {int }},
$$

where

$$
\begin{aligned}
\mathcal{A}_{\text {free }} & =\frac{1}{2} \int_{0}^{\beta} d \tau \sum_{\mathbf{i}} \sum_{a, b=1,2} \psi_{a}\left(\mathbf{x}_{\mathbf{i}}, \tau\right)\left[i \varepsilon_{a b} \partial_{\tau}+Y_{a} \delta_{a b}\right] \psi_{b}\left(\mathbf{x}_{\mathbf{i}}, \tau\right), \\
\mathcal{A}_{\text {int }} & =\mathcal{A}_{\text {int }}^{(2)}+\mathcal{A}_{\mathrm{int}}^{(3)}+\mathcal{A}_{\mathrm{int}}^{(4)}, \\
\mathcal{A}_{\mathrm{int}}^{(2)} & =\frac{1}{2} \int_{0}^{\beta} d \tau \sum_{\mathbf{i}}\left\{\psi_{1}^{2}\left(\mathbf{x}_{\mathbf{i}}, \tau\right)\left[3 U \nu n_{\mathbf{0}}-\Sigma_{\mathrm{cl}}-\Delta_{\mathrm{cl}}\right]\right. \\
& \left.+\psi_{2}^{2}\left(\mathbf{x}_{\mathbf{i}}, \tau\right)\left[U \nu n_{\mathbf{0}}-\Sigma_{\mathrm{cl}}+\Delta_{\mathrm{cl}}\right]\right\}, \\
\mathcal{A}_{\mathrm{int}}^{(3)} & =\frac{1}{2} U \sqrt{2 \nu n_{\mathbf{0}}} \int_{0}^{\beta} d \tau \sum_{\mathbf{i}}\left[\psi_{1}^{3}\left(\mathbf{x}_{\mathbf{i}}, \tau\right)+\psi_{1}\left(\mathbf{x}_{\mathbf{i}}, \tau\right) \psi_{2}^{2}\left(\mathbf{x}_{\mathbf{i}}, \tau\right)\right], \\
\mathcal{A}_{\mathrm{int}}^{(4)} & =\frac{1}{8} U \int_{0}^{\beta} d \tau \sum_{\mathbf{i}}\left[\psi_{1}^{2}\left(\mathbf{x}_{\mathbf{i}}, \tau\right)+\psi_{2}^{2}\left(\mathbf{x}_{\mathbf{i}}, \tau\right)\right]^{2},
\end{aligned}
$$


where

$$
\begin{aligned}
& Y_{1}=-\mu-J z_{0}+\Sigma_{\mathrm{cl}}+\Delta_{\mathrm{cl}}, \\
& Y_{2}=-\mu-J z_{0}+\Sigma_{\mathrm{cl}}-\Delta_{\mathrm{cl}} .
\end{aligned}
$$

The free part of the action, $\mathcal{A}_{\text {free }}$ in Eq. (68), gives rise to the propagator to be used in perturbation expansion. In the momentum representation of the fields Eq. (21), the propagator is given by

$$
G\left(\omega_{n}, \mathbf{q}\right)=\frac{1}{\omega_{n}^{2}+\mathcal{E}^{2}(\mathbf{q})}\left(\begin{array}{lr}
\varepsilon_{\mathbf{q}}+Y_{2} & \omega_{n} \\
-\omega_{n} & \varepsilon_{\mathbf{q}}+Y_{1}
\end{array}\right),
$$

with $\mathcal{E}^{2}(\mathbf{q})=\left(\varepsilon_{\mathbf{q}}+Y_{1}\right)\left(\varepsilon_{\mathbf{q}}+Y_{2}\right)$. To lowest order, one obtains

$$
\Omega=-T \ln Z=-T \ln Z_{0}-T \ln Z_{\text {free }}+T\left\langle\mathcal{A}_{\text {int }}\right\rangle,
$$

where $Z_{0}=e^{-\mathcal{A}_{(0)}}, Z_{\text {free }}=\int \mathcal{D} \psi_{1} \mathcal{D} \psi_{2} e^{-\mathcal{A}_{\text {free }}}=1 / \sqrt{\operatorname{Det} G^{-1}},\left\langle\mathcal{A}_{\text {int }}\right\rangle=\left\{\int \mathcal{D} \psi_{1} \mathcal{D} \psi_{2} \mathcal{A}_{\text {int }} e^{-\mathcal{A}_{\text {free }}}\right\} / Z_{\text {free }}$

Now we evaluate

$$
\begin{aligned}
& \left\langle\psi_{a}^{2}\left(\mathbf{x}_{\mathbf{i}}, \tau\right)\right\rangle=G_{a a}(0)=\frac{\sigma_{a}}{N_{s}}, \quad\left\langle\psi_{a}^{4}\left(\mathbf{x}_{\mathbf{i}}, \tau\right)\right\rangle=\frac{3 \sigma_{a}^{2}}{N_{s}^{2}} \\
& \left\langle\psi_{1}^{2}\left(\mathbf{x}_{\mathbf{i}}, \tau\right) \psi_{2}^{2}\left(\mathbf{x}_{\mathbf{i}}, \tau\right)\right\rangle=\frac{\sigma_{1} \sigma_{2}}{N_{s}^{2}}, \quad\left\langle\mathcal{A}_{\text {int }}^{(3)}\right\rangle=0,
\end{aligned}
$$

with

$$
\sigma_{1}=T \sum_{\mathbf{q}, n} \frac{\varepsilon_{\mathbf{q}}+Y_{2}}{\omega_{n}^{2}+\mathcal{E}^{2}(\mathbf{q})}, \quad \sigma_{2}=T \sum_{\mathbf{q}, n} \frac{\varepsilon_{\mathbf{q}}+Y_{1}}{\omega_{n}^{2}+\mathcal{E}^{2}(\mathbf{q})},
$$

and we find the following thermodynamic potential:

$$
\begin{aligned}
\Omega= & N_{s} \nu n_{\mathbf{0}}\left(-\mu-J z_{0}+\frac{U}{2} \nu n_{\mathbf{0}}\right) \\
& +\frac{1}{2} \sum_{q}\left[\mathcal{E}(\mathbf{q})-\varepsilon(\mathbf{q})+\mu+J z_{0}\right] \\
& +T \sum_{\mathbf{q}} \ln \left(1-e^{-\beta \mathcal{E}(\mathbf{q})}\right)+\frac{U \nu}{8 N}\left[3 \sigma_{1}^{2}+3 \sigma_{2}^{2}+2 \sigma_{1} \sigma_{2}\right] \\
& +\frac{1}{2} \sigma_{1}\left(3 U \nu n_{\mathbf{0}}-Y_{1}-J z_{0}-\mu\right) \\
& +\frac{1}{2} \sigma_{2}\left(U \nu n_{\mathbf{0}}-Y_{2}-J z_{0}-\mu\right),
\end{aligned}
$$

where we have again subtracted $\Omega(T=0, U=0)$.

The parameters $\Sigma_{\mathrm{cl}}$ and $\Delta_{\mathrm{cl}}$ are now determined variationally by requiring that they minimize the thermodynamic potential, i.e., we require $\partial \Omega / \partial \Sigma_{\mathrm{cl}}=0$ and $\partial \Omega / \partial \Delta_{\mathrm{cl}}=0$ [42], or equivalently

$$
\frac{\partial \Omega}{\partial Y_{1}}=0, \quad \frac{\partial \Omega}{\partial Y_{2}}=0
$$

These equations yield

$$
\begin{aligned}
& Y_{1}=3 U \nu n_{\mathbf{0}}-\mu-J z_{0}+\frac{U}{2 N_{s}}\left(3 \sigma_{1}+\sigma_{2}\right), \\
& Y_{2}=U \nu n_{\mathbf{0}}-\mu-J z_{0}+\frac{U}{2 N_{s}}\left(\sigma_{1}+3 \sigma_{2}\right) .
\end{aligned}
$$

The gaplessness of the energy spectrum is now imposed by hand. In fact, by requiring the relation (566), we get from (69) $Y_{2}=0$ which leads to the dispersion

$$
\mathcal{E}(\mathbf{q})=\sqrt{\varepsilon(\mathbf{q})} \sqrt{\varepsilon(\mathbf{q})+2 \Delta},
$$

where $\Delta=Y_{1} / 2$. This leads to the equations

$$
\begin{aligned}
& \Delta=U \nu n_{\mathbf{0}}+\frac{U}{2 N_{s}}\left(\sigma_{1}-\sigma_{2}\right), \\
& \mu+J z_{0}=U \nu n_{\mathbf{0}}+\frac{U}{2 N_{s}}\left(\sigma_{1}+3 \sigma_{2}\right) .
\end{aligned}
$$


Here, we draw the reader's attention to the self-consistency of the HFB approximation as far as the chemical potential is concerned. In fact, the stationary condition $\partial \Omega / \partial n_{\mathbf{0}}=0$ with $\Omega$ given by (74) leads to the following equation for $\mu$ :

$$
\mu+J z_{0}=U \nu n_{\mathbf{0}}+\frac{U}{2 N_{s}}\left(3 \sigma_{1}+\sigma_{2}\right),
$$

which contradicts to $\mu$ of Eq. (78).

To make the theory self-consistent, Yukalov and one of the authors [43] proposed to introduce two chemical potentials: namely, $\mu_{0}$, which corresponds to the Eq. (79), and $\mu_{1}$ corresponding to Eq. (78). Being responsible for subsystem of condensed and uncondensed particles respectively they, naturally, coincide in the normal phase, when $Y_{1}=Y_{2}=0$. In the present work, however, we follow the standard procedure of identifying $\mu$ in (78) as a chemical potential from which we determine the particle densities by differentiation of $\Omega$.

\section{A. The fractions $n_{\mathrm{u}}$ and $\delta$ in VPT}

Applying the well-known relation $N=-\partial \Omega / \partial \mu$ to $\Omega$ in (74) gives

$$
N=N_{s} \nu n_{\mathbf{0}}+\sum_{\mathbf{q}}\left[\frac{(\varepsilon(\mathbf{q})+\Delta) c_{\mathbf{q}}}{\mathcal{E}(\mathbf{q})}-\frac{1}{2}\right] \equiv N_{\mathbf{0}}+N_{\mathbf{u}},
$$

and hence

$$
n_{\mathbf{u}}=\frac{N_{\mathbf{u}}}{N_{s}}=\frac{1}{\nu N_{s}} \sum_{\mathbf{q}}\left[\frac{(\varepsilon(\mathbf{q})+\Delta) c_{\mathbf{q}}}{\mathcal{E}(\mathbf{q})}-\frac{1}{2}\right],
$$

with the $\mathcal{E}(\mathbf{q})$ is the Bogoliubovs dispersion given in (77).

For the anomalous density $\delta$ we obtain

$$
\begin{aligned}
\delta & =\frac{1}{\nu}\left\langle\tilde{\psi}\left(\mathbf{x}_{\mathbf{i}}, \tau\right) \tilde{\psi}\left(\mathbf{x}_{\mathbf{i}}, \tau\right)\right\rangle=\frac{1}{2 N_{s} \beta \nu}\left[G_{11}(0)-G_{22}(0)\right] \\
& =\frac{\left(\sigma_{1}-\sigma_{2}\right)}{2 N_{s} \nu}=-\frac{\Delta}{\nu N_{s}} \sum_{\mathbf{q}} \frac{c_{\mathbf{q}}}{\mathcal{E}(\mathbf{q})},
\end{aligned}
$$

where we used Eqs. (70) and (73).

Using now (82) in (78) gives the equation:

$$
\Delta=U \nu\left(n_{0}+\delta\right),
$$

which is formally the same as the one in before (54) with (58). The only difference between these two approximations is in the sign of anomalous density, which is, in general, $\delta>0$ in the collective quantum field theory and $\delta<0$ in HFB.

Summarizing we collect here the main equations in both approximations:

$$
\begin{aligned}
\Delta & =U \nu\left(n_{\mathbf{0}}+\delta\right), \quad n_{\mathbf{0}}=1-n_{\mathbf{u}}, \\
\delta & =\xi \frac{\Delta}{\nu N_{s}} \sum_{\mathbf{q}} \frac{c_{\mathbf{q}}}{\mathcal{E}(\mathbf{q})}, \\
\mathcal{E}(\mathbf{q}) & =\sqrt{\varepsilon(\mathbf{q})} \sqrt{\varepsilon(\mathbf{q})+2 \Delta}, \\
c_{\mathbf{q}} & =\frac{1}{2}+\frac{1}{e^{\beta \mathcal{E}(\mathbf{q})-1}}, \\
\mu & =2 U \nu-\Delta-J z_{0}, \\
\xi & =\left\{\begin{array}{l}
-1, \text { HFB } \\
+1, \text { Two Collective Quantum Fields and LOAF },
\end{array}\right.
\end{aligned}
$$

where $n_{\mathbf{u}}$ is given by (81).

Note that similar relations hold for atomic gases. A difference occurs for the $T>T_{c}$ phase. There one may use replacements listed in Appendix B. In fact, in the normal phase, $n_{\mathbf{0}}=0$, HFB theory gives

$$
\Delta=U \nu \delta=-\frac{\Delta}{\nu N_{s}} \sum_{\mathbf{q}} \frac{c_{\mathbf{q}}}{\mathcal{E}(\mathbf{q})} .
$$


Since the right-hand-side of this equation is positive, while the left-hand-side is negative, at least for optical lattices, Eq. (90) has the only solution $\Delta=0$. This means that in the normal phase $n_{\mathbf{0}}=0$ and $\delta=0$ [see Eq. (85)] simultaneously. Therefore HFB theory does not predict a superfluid phase without a condensate, thus being in contrast to the two-collective quantum field result of Cooper et.al. in Ref. [23] at the mean-field level.

From above discussions it is easy to understand that VPT gives no shift in $T_{c}$ due to interaction. In fact, when $T \rightarrow T_{c}$, the condensed fraction $n_{\mathbf{0}} \rightarrow 0$, and hence $\Delta \rightarrow 0$. The expression for $n_{\mathbf{u}}$, will coincide with that for the ideal gas, i.e., Eq. (81) becomes

$$
\nu=\frac{1}{N_{s}} \sum_{\mathbf{q}} \frac{1}{e^{\beta \varepsilon(\mathbf{q})}-1} \equiv \frac{1}{N_{s}} \sum_{\mathbf{q}} \frac{1}{e^{\varepsilon(\mathbf{q}) / T_{c}^{0}}-1},
$$

which means that $T_{c}=T_{c}^{0}$ for HFB and, hence, $\Delta T_{c}=T_{c}-T_{c}^{0}=0$.

\section{RESULTS AND DISCUSSION}

\section{A. Quantum phase transition in two-Collective Quantum Field Theory and VPT}

First we discuss the existence of QPT in optical lattices for two collective quantum fields at the mean field level and for the HFB approximation. It has been shown that for dilute atomic Bose gases Collective Quantum Field approximation does not predict QPT 20] while HFB does [39]. Below we show that in the case of $3 d$ optical lattices the situation is vice-versa. This can be understood in the following way. Lets rewrite the main equation at $T=0$ as:

$$
n_{\mathbf{0}}(\Delta)=\frac{\Delta}{U \nu}-\delta(\Delta)
$$

It is clear that for interacting system, $U \neq 0$ and $\Delta \neq 0$. Since in the collective quantum field theory $\delta(\Delta)>0$, the Eq. (92) may have solution $n_{\mathbf{0}}(\Delta)=0$ with $\Delta \neq 0$ (see Table I). However, in HFB approximation $\delta(\Delta)<0$ and $n_{0}(\Delta)$ in (92) may have the only solution as $n_{\mathbf{0}}>0$ for $\Delta \neq 0$. Note that in the case of dilute atomic gases at $T=0$ [44]

$$
\begin{aligned}
& \delta(\Delta)=-8 \rho \sqrt{\gamma / \pi}<0 \quad \text { Two-Collective Quantum Field } \\
& \delta(\Delta)=+8 \rho \sqrt{\gamma / \pi}>0 \quad \text { HFB }
\end{aligned}
$$

with the dimensionless gas parameter $\gamma=a_{s}^{3} \rho$ that characterizes the interaction strength of the gas after renormalization. It is formed from the $s$-wave scattering length $a_{s}$ and the particle density $\rho$. This sign change is responsible for the dilute atomic gases has a QPT in the HFB approximation, but not in the two-collective quantum field theory at the mean-field level. In Fig. 1, the condensed fraction $n_{0}$ as a function of $u=U / J$ is presented for $\nu=1,2,3,4$. This may be compared with $u_{\text {crit }}=6(\sqrt{\nu}+\sqrt{\nu+1})^{2}$ given in Gutzwiller's approximation. It is seen that although the two - collective quantum field theory predicts rather large value for $u_{\text {crit }}$ (see Table I), it gives desirable second order phase transition.

\section{B. Critical temperature $T_{c}^{0}$ for ideal cases}

Before we study the shift of $T_{c}$, let us estimate the critical temperature $T_{c}^{0}$ for the free optical lattice with $U=0$. Assuming $\Delta=0$ in Eq. (63), we obtain the well-known formula

$$
\nu=\int_{0}^{1} d q_{1} d q_{2} d q_{3} \frac{1}{e^{\varepsilon_{q} / T_{c}^{0}}-1} .
$$

Introducing dimensionless parameters $t_{c}^{0}=T_{0}^{c} / J, \quad \hat{\varepsilon}_{\mathbf{q}}=\varepsilon_{\mathbf{q}} / 2 J=\sum_{\alpha=1}^{3}\left(1-\cos \pi q_{\alpha}\right)$, we may rewrite (94) as

$$
\nu=\int_{0}^{1} d q_{1} d q_{2} d q_{3} \frac{1}{e^{2 \hat{\varepsilon} \mathbf{q} / t_{c}^{0}}-1}
$$

which can be considered as a nonlinear equation for $t_{c}^{0}$ at a given filling factor $\nu$. Our numerical estimations for $t_{c}^{0}$ are given in Table I. It is seen that for $\nu=1, T_{c}^{0}=5.6 \mathrm{~J}$, which is in consistent with other estimates given in the references [3, 33]. 


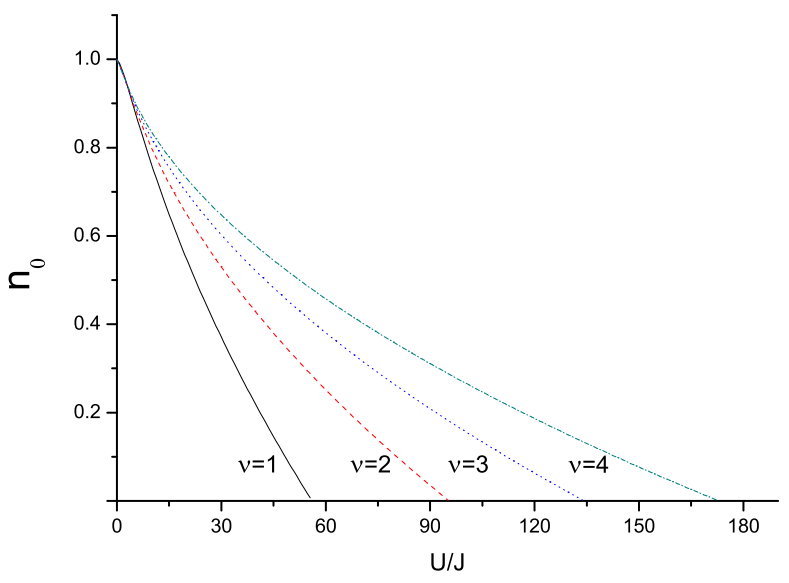

FIG. 1: (Color online) The condensed fraction $n_{0}$ at zero temperature as a function of $u=U / J$ for various filling factors, $\nu$. It is seen that $n_{0}$ goes to zero smoothly and vanishes at $u_{\text {crit }}$. This may be compared with following results by Gutzwiller's approximation: $u_{\text {crit }}(\nu=1)=34.97, u_{\text {crit }}(\nu=2)=59.39, u_{\text {crit }}(\nu=3)=83.56, u_{\text {crit }}(\nu=4)=107.66$.

TABLE I: Critical parameters of Bose-Hubbard model vs. filling factor $\nu$ in the two collective quantum field approach. $u_{c}=(U / J)_{c}$ is given in the second row. The critical temperatures of ideal optical lattices in $d=3$ are listed in units $J$ in the third row. The forth row presents approximated values of $t_{c}^{0}$ [see Eq. (96)]

\begin{tabular}{|c|c|c|c|c|c|}
\hline$\nu$ & 1 & 2 & 3 & 4 & 5 \\
\hline$u_{c}=(U / J)_{c}$ & 56.08 & 95.4 & 134.3 & 173 & 211.7 \\
\hline$t_{c}^{0}=T_{c}^{0} / J$ & 5.6 & 9.69 & 13.70 & 17.70 & 21.67 \\
\hline$t_{c}^{\mathrm{O}}$ in small $q$ approximation & 5.06 & 10.07 & 15.2 & 20.25 & 25.32 \\
\hline
\end{tabular}

Note that $T_{c}^{0}$ can be approximated as $T_{c}^{0}=5.6 J \nu^{0.825}$ in the range $\nu \in(1,5)$ including also non integer values. In the third row of Table I an approximated values of $t_{c}^{0}$ are presented. This approximation, say, spherical approximation at small momentum, is obtained by following replacements in (95):

$$
\begin{aligned}
& \int_{0}^{1} d q_{1} d q_{2} d q_{3} f(\mathbf{q}) \rightarrow \frac{\pi}{2} \int_{0}^{q_{d}} q^{2} d q f(q), \\
& \hat{\varepsilon}_{\mathbf{q}} \rightarrow \frac{\pi^{2}}{2} \mathbf{q}^{2}, \quad\left(e^{\varepsilon_{q} / T_{c}^{0}}-1\right)^{-1} \rightarrow \frac{T_{c}^{0}}{\varepsilon(\mathbf{q})},
\end{aligned}
$$

where the Debye momentum $q_{\mathrm{D}}$ defined by the equation:

$$
1=\int_{0}^{1} d q_{1} d q_{2} d q_{3}=\frac{\pi}{2} \int_{0}^{q_{d}} q^{2} d q,
$$

equals to $q_{\mathrm{d}}=(6 / \pi)^{1 / 3} \approx 1.24$ for $d=3$. This gives $T_{c}^{0} / J=2 \nu \pi(\pi / 6)^{1 / 3}$. It is seen that this approximation works with roughly $10 \%$ accuracy for $\nu \leq 3$.

\section{The shift in $T_{c}$ caused by the interaction}

We are now prepared to estimate the shift $\Delta T_{c} / T_{c}^{0}=\left(T_{c}-T_{c}^{0}\right) / T_{c}^{0}$ analytically. Above we have shown that the shift $\Delta T_{c} / T_{c}^{0}=0$ for VPT or equivalently for HFB. For LOAF the integrals in the main equations are dominated by small momenta. At $T \rightarrow T_{c}$ for $n_{\mathbf{0}}=0, n_{\mathbf{u}}=1$ they are given by

$$
\Delta=U \Delta \int_{0}^{1} d q_{1} d q_{2} d q_{3} \frac{f_{B}(\mathcal{E}(\mathbf{q}))}{\mathcal{E}(\mathbf{q})}
$$




$$
1=\frac{1}{\nu} \int_{0}^{1} d q_{1} d q_{2} d q_{3} \frac{\left(\varepsilon_{\mathbf{q}}+\Delta\right)}{\mathcal{E}(\mathbf{q})} f_{B}(\mathcal{E}(\mathbf{q}))
$$

with $\mathcal{E}(\mathbf{q})=\sqrt{\varepsilon_{\mathbf{q}}} \sqrt{\varepsilon_{\mathbf{q}}+2 \Delta}, \quad f_{B}(\mathcal{E}(\mathbf{q}))=1 /\left(e^{\beta_{c} \mathcal{E}(\mathbf{q})}-1\right), \quad \beta_{c}=1 / T_{c}$

Note that in (98) we may assume $\Delta \neq 0$ and divide both sides of (98) by $\Delta$. The critical temperature of ideal gas $T_{c}^{0}$ is the solution of Eq. (99) with $\Delta=0$, i.e.,

$$
1=\frac{1}{\nu} \int_{0}^{1} \frac{d q_{1} d q_{2} d q_{3}}{e^{\varepsilon_{\mathbf{q}} / T_{c}^{0}}-1}
$$

Now we introduce dimensionless variables:

$$
\begin{aligned}
& \Delta=u^{2} \kappa^{2} T_{c}^{0}, \quad T_{c}=T_{c}^{0} \alpha, \quad T_{c}^{0}=J t_{c}^{0}, \\
& \varepsilon_{\mathbf{q}}=2 J \hat{\varepsilon}_{\mathbf{q}}, \quad \mathcal{E}(\mathbf{q})=2 J \hat{\mathcal{E}}(\mathbf{q}),
\end{aligned}
$$

with $\hat{\varepsilon}_{\mathbf{q}}=\sum_{\alpha}\left(1-\cos \pi \mathbf{q}_{\alpha}\right), \quad \hat{\mathcal{E}}(\mathbf{q})=\sqrt{\hat{\varepsilon}_{\mathbf{q}}} \sqrt{\hat{\varepsilon}_{\mathbf{q}}+u^{2} \kappa^{2} t_{c}^{0}}, \quad \Delta T_{c} / T_{c}^{0}=\alpha-1$ and $t_{c}^{0}$ are given in the third row of Table I.

The scaled equations can be rewritten as follows:

$$
\begin{aligned}
& 0=1-\frac{u}{2} \int_{0}^{1} \frac{f_{B}(\hat{\mathcal{E}}(\mathbf{q})) d q_{1} d q_{2} d q_{3}}{\hat{\mathcal{E}}(\mathbf{q})} \\
& 0=1-\frac{1}{\nu} \int_{0}^{1} d q_{1} d q_{2} d q_{3} \frac{\hat{\mathcal{E}}_{\mathbf{q}}+u^{2} \kappa^{2} t_{c}^{0} / 2}{\hat{\mathcal{E}}(\mathbf{q})} f_{B}(\hat{\mathcal{E}}(\mathbf{q})),
\end{aligned}
$$

with $f_{B}(\hat{\mathcal{E}}(\mathbf{q}))=1 /\left(e^{2 \hat{\mathcal{E}}(\mathbf{q}) / \alpha t_{c}^{0}}-1\right)$

Bearing in mind (100), we may rewrite (103) as

$$
\int_{0}^{1} d q_{1} d q_{2} d q_{3}\left\{\frac{1}{e^{2 \hat{\mathcal{E}}(\mathbf{q}) / t_{c}^{0}}-1}-\frac{\hat{\varepsilon}_{\mathbf{q}}+u^{2} \kappa^{2} t_{c}^{0} / 2}{\hat{\mathcal{E}}(\mathbf{q})\left(e^{2 \hat{\mathcal{E}}(\mathbf{q}) / \alpha t_{c}^{0}}-1\right)}\right\}=0 .
$$

The nonlinear equations (102) and (104) should be solved with respect to $\kappa$ and $\alpha$ with given numbers $u=U / J$ and $t_{c}^{0}$. To do this we make replacements (96). Then Eqs. (102) and (104) can be rewritten as

$$
\begin{aligned}
& 1-\frac{u \alpha t_{c}^{0}}{4 \sqrt{2} \pi^{2}} \int_{0}^{\varepsilon_{\mathrm{D}}} \frac{d \varepsilon}{\sqrt{\varepsilon}\left(\varepsilon+u^{2} \kappa^{2} t_{c}^{0}\right)}=0, \\
& \int_{0}^{\varepsilon_{\mathrm{D}}} \frac{d \varepsilon}{\sqrt{\varepsilon}}\left\{1-\frac{\alpha\left(\varepsilon+u^{2} \kappa^{2} t_{c}^{0} / 2\right)}{\varepsilon+u^{2} \kappa^{2} t_{c}^{0}}\right\}=0,
\end{aligned}
$$

where $\varepsilon_{\mathrm{D}}=\pi^{2} q_{\mathrm{D}}^{2} / 2=\left(\pi^{2} / 2\right)(6 / \pi)^{2 / 3}$

The integrals in (105) and (107) are easily done and yield

$$
\begin{aligned}
& 0=\sqrt{2}\left(6 \pi^{2}\right)^{1 / 3}(1-\alpha)+u \alpha \kappa \sqrt{t_{c}^{0}} \arctan \tilde{\theta} \\
& 0=4 \pi^{2} \kappa-\sqrt{2} \alpha \sqrt{t_{c}^{0}} \arctan \tilde{\theta}
\end{aligned}
$$

where $\tilde{\theta}=\sqrt{2}\left(6 \pi^{2}\right)^{1 / 3} /\left(2 \kappa u \sqrt{t_{c}^{0}}\right)$. Excluding $\alpha$ from (110) and inserting it to (108) gives

$$
\begin{aligned}
\alpha & =\frac{2 \sqrt{2} \pi^{2} \kappa}{\sqrt{t_{c}^{0}} \arctan \tilde{\theta}} \\
0 & =4 \kappa \pi^{8 / 3} 6^{1 / 3}-\sqrt{2 t_{c}^{0}}\left[\left(6 \pi^{2}\right)^{1 / 3}+2 u \kappa^{2} \pi^{2}\right] \arctan \tilde{\theta}
\end{aligned}
$$

Now we consider separately two regimes: 
a) Weak interacting regime. Expanding (111) and (112) in linear order by $u$ we get

$$
\begin{aligned}
\alpha & =\frac{4 \pi^{2} \kappa \sqrt{2}}{\sqrt{t_{c}^{0}}}+\frac{8 \kappa^{2} u}{3}\left(\frac{6}{\pi}\right)^{2 / 3}, \\
\kappa & =\frac{\sqrt{2 t_{c}^{0}}}{8 \pi} .
\end{aligned}
$$

Now inserting $\kappa$ into (113) we finally obtain

$$
\alpha=1+\frac{u t_{c}^{0}}{12}\left(\frac{6}{\pi^{4}}\right)^{2 / 3}+O\left(u^{2}\right)
$$

and hence

$$
\frac{\Delta T}{T_{c}^{0}}=\alpha-1=\frac{u t_{c}^{0}}{12}\left(\frac{6}{\pi^{4}}\right)^{2 / 3}+O\left(u^{2}\right)
$$

which means that for small coupling constant, i.e. $(U / J)<1$, the shift is positive and increases with $U / J$.

b) Strong interacting regime. In this region, $\Delta / u^{2}$ and hence, $\kappa$ is small, so we may use a linear approximation in $\kappa$ in Eqs. (111), (112)

$$
\begin{aligned}
\alpha & =\frac{4 \pi^{2} \kappa \sqrt{2}}{\sqrt{t_{c}^{0}}}, \\
0 & =\frac{\sqrt{2 t_{c}^{0}}\left(6 \pi^{5}\right)^{1 / 3}}{2}-\kappa\left[2 u t_{c}^{0}+4\left(6 \pi^{8}\right)^{1 / 3}\right] .
\end{aligned}
$$

This leads to following equation

$$
\alpha=\frac{2 \pi^{8 / 3} 6^{1 / 3}}{u t_{c}^{0}+2 \pi^{8 / 3} 6^{1 / 3}}=\frac{T_{c}}{T_{c}^{0}}
$$

from which one may conclude that $T_{c}$ decreases with increasing $u$, i.e.

$$
\frac{\Delta T_{c}}{T_{c}^{0}}=\alpha-1=-\frac{u t_{c}^{0}}{u t_{c}^{0}+2 \pi^{8 / 3} 6^{1 / 3}}<0 .
$$

Thus, our analytical estimate shows that the critical temperature $T_{c}$ as a function of the coupling constant $U$, i.e. the function $T(u)$ first increases and then decreases with increasing $u$ for optical lattices. The suppression of $T_{c}$ at large coupling constant is in agreement with experimental measurements [4] .

In Fig. 2 we present $T_{c}$ (in unit of $J$ ) vs. $u$ for $\nu=1$. The solid line correspond to the exact numerical calculation, i.e., the numerical solutions of Eqs. (98), (99). The experimental points (circles) are taken from [4], solid diamonds are from Monte-Carlo calculations taken from ref. [3] The suppression of $T_{c}$ at large coupling constant is found for integer $\nu \geq 1$ also, as it is seen in Fig. 3 .

In Fig. 4 we present the critical values of the self energy $\Delta_{c}=\Delta\left(T=T_{c}\right)$ in units $J$ vs. $(U / J)$. Observe that when $J$ is fixed, $\Delta_{c}$ increases with increasing $u$ and $\nu$. On the other hand we observed that $\Delta_{c}$ in units $T_{c}^{0}(\nu)$, i.e. $\Delta_{c}\left(T=T_{c}\right) / T_{c}^{0}(\nu)$ vs. $u$ is almost independent on $\nu$, e.g., $\left.\left(\Delta_{c} / T_{c}^{0}\right)\right|_{\nu=1}=7.656$ and $\left.\left(\Delta_{c} / T_{c}^{0}\right)\right|_{\nu=4}=7.780$ at $u=42.0$.

Now we consider the behavior of $\Delta$ for $T>T_{c}$. It was suggested by Cooper et.al. [23] that in the temperature range $T \in\left(T_{c}, T^{*}\right)$ there exists a $U(1)$-symmetric phase with $n_{\mathbf{0}}=0$ but $\delta \neq 0$. This would imply the existence of a superfluid state without a condensate. However, by solving (63) for $\Delta$ and $\varphi^{\prime}$, we could not find, for optical lattices, any solution with $\Delta \neq 0, \varphi^{\prime} \neq 0$. Instead, the equations for $T>T_{c}$, have a solution with $\Delta=0, \varphi^{\prime}=2 U \nu-J z_{0}-\mu$. In this normal state with $\delta=0$, the filling factor that characterizes the particle density, is determined by the well-known equation

$$
\begin{aligned}
\nu & =\frac{1}{N_{s}} \sum_{\mathbf{q}} \frac{1}{e^{\beta\left(\varepsilon \mathbf{q}^{\left.-2 U \nu-J z_{0}-\mu\right)}-1\right.}} \\
& =\int_{0}^{1} d q_{1} d q_{2} d q_{3} \frac{1}{e^{\beta\left(\varepsilon \mathbf{q}^{\left.-2 U \nu-J z_{0}-\mu\right)}-1\right.}}
\end{aligned}
$$

with the bare dispersion $\varepsilon_{\mathbf{q}}=2 J \sum_{\alpha=1}^{3}\left(1-\cos \pi q_{\alpha}\right)$.

The chemical potential of interacting bosons in $T>T_{c}$ may be evaluated self consistently from Eq. (121) with input parameters $\nu, J, U$, and $T$, or given by an external field (pumping) as in the case of triplons [24, 25]. 


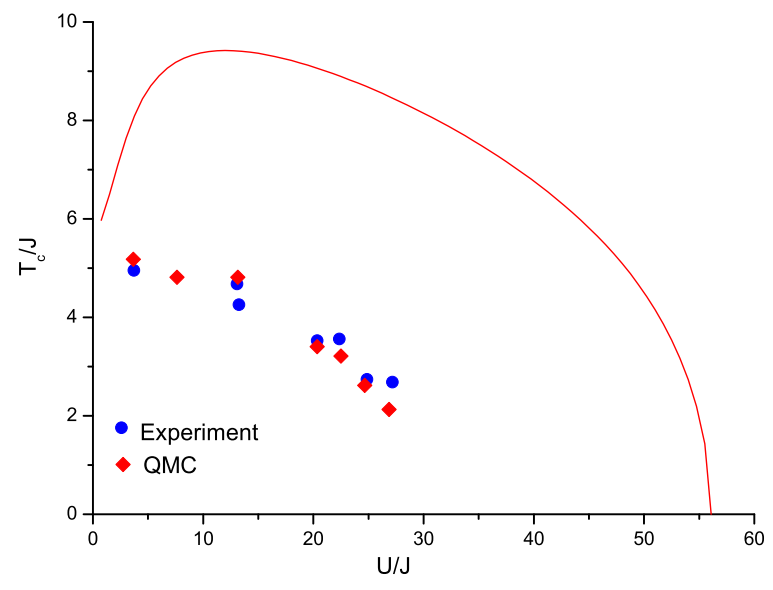

FIG. 2: (Color online) Behavior of $T_{c}$ (in units $\mathrm{J}$ ) as a function of $U / J$ in the saddle-point approximation to the two-collective field theory for $\nu=1$. The circles show experimental values given in [4], solid diamonds are from Monte-Carlo calculations of Ref. [3]. Note the initial rise that was found also in atomic gases in Ref. [22].

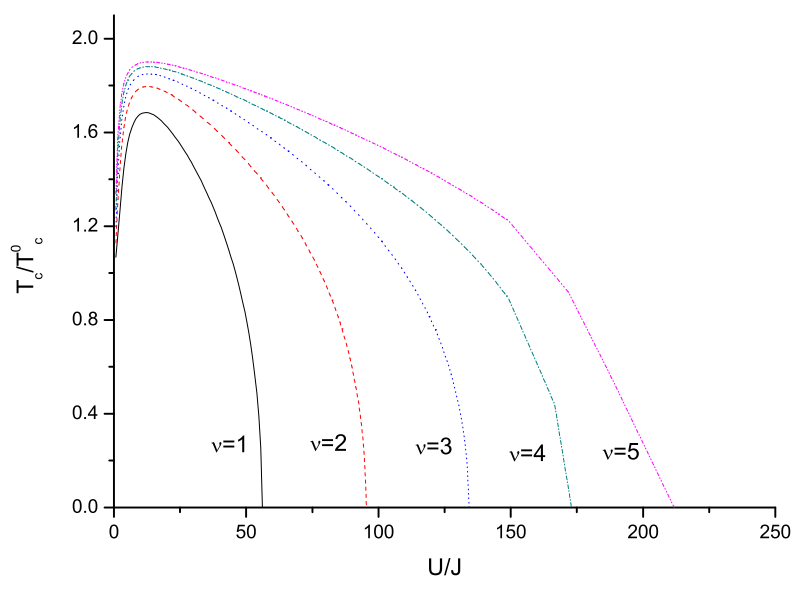

FIG. 3: (Color online) The same curves as in Fig. 1 but for $\nu=1,2,3,4,5$.

\section{CONCLUSION}

In this paper we have developed a Collective Quantum Field Theory and a Variational Perturbation Theories for $d=3$ optical lattices at very low temperatures. Both approximations satisfy Hugenholtz-Pines theorem. We have shown that, a two-Collective Quantum Field treatment in the saddle point approximation predicts a second - order Quantum Phase transition, that is missed in the VPT [45]. Unfortunately, the predicted critical value of $(U / J)_{c}$ e.g. for $\nu=1$ is nearly twice as large as the experimental one. Note that the main equations of the previously mentioned approximation LOAF [19] (recall page 2) and VPT are formally the same. The difference is in the sign of the anomalous density $\delta$, as it is seen from equations (84)- (899). We obtained analytical estimation for the shift of critical temperature $T_{c}$ due to the point interaction both in the weak and strong interaction regimes. It is zero for VPT, while it has a nontrivial dependence on the coupling strength $(U / J)$ in the Collective Quantum Field treatment as well as in the LOAF approximation. The general behavior of the phase diagram compares qualitatively well with existing experimental and ab initio quantum Monte Carlo results. The similar behavior e.g. suppression of the 


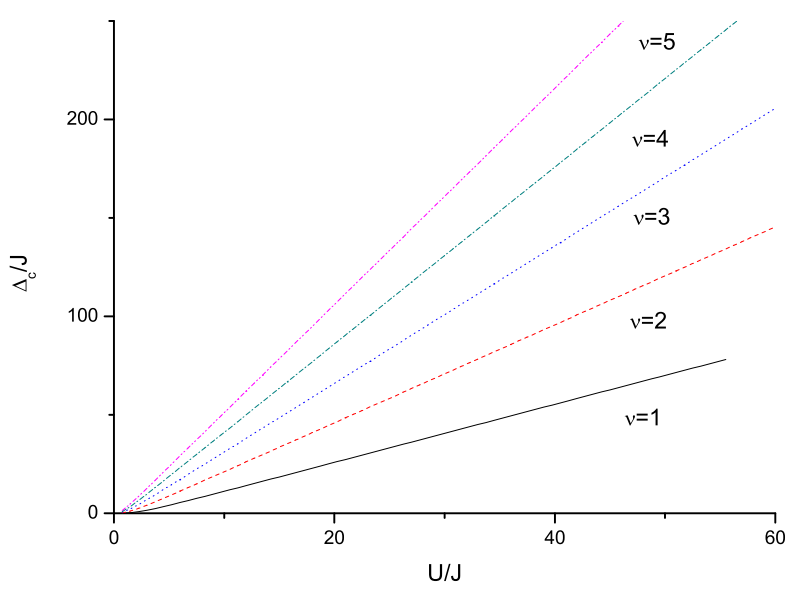

FIG. 4: (Color online) The critical $\Delta_{c}$ vs. $u$ for various filling factors $\nu$.

critical temperature at large gas parameter for homogenous interacting Bose gases have also found in Path - Integral Monte - Carlo simulations [46]. As to the dependence of the critical temperature on the filling factor, $T_{c} / T_{c}^{0}$ increases with increasing $\nu$ at fixed $U / J$. From figures Fig. 1 and Fig. 2 one may conclude that in order to describe the phase transitions in optical lattices more accurately, the present theory should be extended beyond the saddle point approximation used in Eq. (31), or in the spirit of B-DMFT [15]. We have found no exotic superfluid state with finite anomalous density but zero condensate. Therefore, the temperatures $T^{*}$ and $T_{c}$ introduced by Cooper et.al. 23] coincide. The system is in superfluid state for $0 \leq T \leq T_{c}$, and in normal state for $T>T_{c}$. It is natural that the condensation will always be present in the one-body channel (see Eq. (13)).

\section{Acknowledgments}

The work is supported in part by DAAD and Uzbek Science Foundation. We are indebted to Fred Cooper for useful discussions. 


\section{Appendix A}

Below we derive Hugenholtz-Pines theorem

$$
\Sigma_{\mathrm{cl}}-\Delta_{\mathrm{cl}}=\mu+J z_{0},
$$

of Sect. II for optical lattices. The normal $\Sigma_{\mathrm{cl}}$,and anomalous $\Delta_{\mathrm{cl}}$ self-energies in (122) correspond to the normal $G_{\mathrm{n}}\left(r, r^{\prime}\right)=\left\langle T_{\tau} \tilde{\psi}(r) \tilde{\psi}^{+}\left(r^{\prime}\right)\right\rangle$ and anomalous $G_{\mathrm{an}}\left(r, r^{\prime}\right)=-\left\langle T_{\tau} \tilde{\psi}(r) \tilde{\psi}\left(r^{\prime}\right)\right\rangle$ Green functions respectively. In the Cartesian parametrization of the quantum field (16) we have:

$$
\begin{aligned}
\Sigma_{\mathrm{cl}} & =\frac{1}{2}\left[\Pi_{11}+\Pi_{22}\right], \\
\Delta_{\mathrm{cl}} & =\frac{1}{2}\left[\Pi_{22}-\Pi_{11}\right],
\end{aligned}
$$

where $\Pi_{a b}$ are defined by Dyson-Beliaev equations [47]:

$$
\left(\hat{G}^{-1}\right)_{a b}-\left(\hat{G}_{0}^{-1}\right)_{a b}=\Pi_{a b},
$$

and the Green function $\hat{G}_{0}$ corresponds to the noninteracting situation

$$
G_{0}^{-1}\left(\omega_{n}, \mathbf{q}\right)=\left(\begin{array}{lr}
\varepsilon(\mathbf{q})-\mu-J z_{0} & -\omega_{n} \\
\omega_{n} & \varepsilon(\mathbf{q})-\mu-J z_{0}
\end{array}\right) .
$$

The interacting Green function $\hat{G}^{-1}$ is defined in Eq.(24). Using (20), (33), (24), (126) in (125) gives:

$$
\begin{aligned}
& \Pi_{11}=X_{1}+\mu=\cosh \theta \varphi_{0}-\Delta, \\
& \Pi_{22}=X_{2}+\mu=\cosh \theta \varphi_{0}+\Delta, \\
& \Pi_{12}=\Pi_{21}=0 .
\end{aligned}
$$

Inserting (127) into (123) and (124) one derives

$$
\begin{aligned}
& \Sigma_{\mathrm{cl}}=\varphi_{0} \cosh \theta, \\
& \Delta_{\mathrm{cl}}=\Delta .
\end{aligned}
$$

and hence

$$
\Sigma_{\mathrm{cl}}-\Delta_{\mathrm{cl}}=\varphi_{0} \cosh \theta-\Delta=\varphi^{\prime}+\mu+J z_{0}-\Delta,
$$

where we have used Eq.(33). As it has been shown in Sect. II, in the condensed phase $\varphi^{\prime}=\Delta$ and Eq. (129) becomes equivalent to the Hugenholtz-Pines theorem, i.e. to Eq.(122).

The relation (122) in HFB approximation can be proved in a similar way. 


\section{Appendix B}

Here we present formal equivalence between Bose-Hubbard Hamiltonian (1) in Wannier representation and standard Hamiltonian for homogeneous dilute atomic gases

$$
H=\int d \mathbf{r} \Psi^{\dagger}(\mathbf{r})\left[-\frac{\vec{\nabla}^{2}}{2 m}-\mu\right] \Psi(\mathbf{r})+\frac{g}{2} \int d \mathbf{r}\left[\Psi^{\dagger}(\mathbf{r}) \Psi(\mathbf{r})\right]^{2},
$$

where $g$ is the constant of contact interatomic interaction. Using the replacements listed in Table II we obtain for $\Omega$ and the extremality equations in dilute atomic gases versus optical lattices the relevant quantitied as derived in Sections II and III. Of course, an appropriate renormalization procedure is implied in dilute atomic gases.

TABLE II: Formal similarity between Hamiltonians (11) and (130)

\begin{tabular}{|l|c|c|c|}
\hline Quantity & Homogeneous atomic gases & $3 D$ Bose-Hubbard model & Comment \\
\hline Volume & $V$ & $N_{s}$ & $N_{s}-$ number of sites \\
\hline Density & $\rho=N / V$ & $\varepsilon=N / N_{s}$ & $\nu-$ filling factor \\
\hline Bare dispersion & $\varepsilon(\mathbf{q})=\mathbf{q}^{2} / 2 m$ & No additional magnetic trap \\
\hline Chemical potential & $\mu$ & $\mu J \sum_{\alpha=1}^{3}\left(1-\cos \pi q_{\alpha}\right)$ & $N=-\left(\frac{\partial \Omega}{\partial \mu}\right)_{T}$ \\
\hline Momentum summation & $\frac{1}{V} \sum_{q} f(\varepsilon(\mathbf{q}))=\frac{1}{2 \pi^{2}} \int_{0}^{\infty} q^{2} d q f(\varepsilon(q))$ & $\frac{1}{N_{s}} \sum_{q} f(\varepsilon(\mathbf{q}))=\int_{0}^{1} d q_{1} d q_{2} d q_{3} f(\varepsilon(q))$ & $d=3$ \\
\hline Normalization of densities & $\rho_{0}+\rho_{1}=\rho$ & $n_{0}+n_{1}=1$ & $\begin{array}{c}\text { In the condensed phase. } \\
\text { No disorder. }\end{array}$ \\
\hline
\end{tabular}

[1] O. Morsch and M. Oberthaler Rev. Mod. Phys. 78, 179 (2006).

[2] R. Raussendorf, D.E. Browne, and H.J. Briegel, Phys. Rev. A 68, 022312 (2003).

[3] B. Capogrosso-Sansone, N.V. Prokofev, and B.V. Svistunov, Phys. Rev. B 75, 134302 (2007).

[4] Trotzky, L. Pollet, F. Gerbier, U. Schnorrberger, I. Bloch, N.V. Prokofev, B. Svistunov and M. Troyer, Nature Phys. 6, 998 (2010).

[5] H.T.C. Stoof, K.B. Gubbels, and D.B.M. Dickerscheid, Ultracold Quantum Fields (Springer, 2009).

[6] M. Lewenstein, A. Sanpera, and V. Ahufinger, Ultracold atoms in optical lattices: Simulating quantum many-body systems (Oxford University Press, 2012).

[7] M. Ueda, Fundamentals and new frontiers of Bose- Einstein condensation (World Scientific, Singapore, 2010).

[8] J.K. Freericks, H.R. Krishnamurthy, Yasuyuki Kato, Naoki Kawashima, and Nandini Trivedi, Phys. Rev. A 79, 053631-1-22 (2009).

[9] F.E.A. dos Santos and A. Pelster, Phys. Rev. A 79, 013614 (2009).

[10] A. Dutta, C. Trefzger, and K. Sengupta, arXiv:1111.5085v3 (2012).

[11] D.-S. Lühmann, Phys. Rev. A 87, 043619 (2013).

[12] L. Amico and V. Penna, Phys. Rev. Lett. 80, 2189-2192 (1998).

[13] P. Buonsante and A. Vezzani, Phys. Rev. A 70, 033608 (2004).

[14] K. Byczuk and D. Vollhardt Phys. Rev. B 77, 235106 (2008).

[15] P. Anders et al., New J. Phys. 13, 075013 (2011)

[16] A. Rancon and N. Dupuis Phys. Rev. A 86, 043624 (2012)

[17] D. van Oosten, P. van der Straten, and H.T.C. Stoof Phys. Rev. A 63, 053601 (2001).

[18] H. Kleinert, Z. Narzikulov, and Abdulla Rakhimov, Phys. Rev. A 85, 063602 (2012).

[19] F. Cooper, B. Mihaila, J.F. Dawson, C.C. Chien, and E. Timmermans, Phys. Rev. A 83, 053622 (2011).

[20] B. Mihaila, F. Cooper, J.F. Dawson, C.C. Chien, and E. Timmermans, Phys. Rev. A 84, 023603 (2011).

[21] G. Baym, J.-P.Blaizot, M.Holzmann, F. Laloe, and D.Vautherin, Phys. Rev. Lett. 83, 1703 (1999).

[22] H. Kleinert, Mod. Phys. Lett. B 17, 1011 (2003) (klrt.de/320).

[23] J.F. Dawson, B. Mihaila, and F. Cooper, Phys. Rev. A 86, 013603 (2012);

J.F. Dawson, F. Cooper, C.-C. Chien, and B. Mihaila, Phys. Rev. A 88, 023607 (2013).

[24] Abdulla Rakhimov, S. Mardonov, and E. Ya. Sherman, Annals of Phys. 326, 2499 (2011).

[25] Abdulla Rakhimov, S. Mardonov, E. Ya. Sherman, and A. Schilling, New J. Phys. 14, 113010 (2012).

[26] For field theories on a lattice see H. Kleinert, Gauge Fields in Condensed Matter, Vol. I Superflow and Vortex Lines, World Scientific, Singapore 1989 (klnrt.de/b1). 
[27] H. Kleinert, Fortschr. Phys. 26, 565 (1978) (klnrt.de/55).

[28] H. Kleinert, Collective Classical an Quantum Fields, World Scientific, Singapore, 2013 (klnrt.de/b7).

[29] H. Kleinert, Fortschr. Phys. 30, 187 (1982).

[30] R.P. Feynman and H. Kleinert, Phys. Rev. A 34, 5080 (1986).

[31] H. Kleinert and Schulte-Frohlinde, Critical Properties of $\Phi^{4}$-Theories, World Scientific, Singapore 2001 (klnrt.de/b8).

[32] H. Kleinert, EJTP 8, 15 (2011) (klnrt.de/387).

[33] V. I. Yukalov, Laser Physics 19, 1 (2009).

[34] H. Kleinert, EJTP 8, 25 (2011) (klnrt.de/391).

[35] I. Danshita and P. Naidon, Phys. Rev. A 79, 043601 (2009).

[36] See Section 4.3 in Ref. 37].

[37] H. Kleinert, Path Integrals in Quantum Mechanics, Statistics and Polymer Physics, World Scientific Publishing Co., Singapore 1995 (klnrt.de/b5). See the discussion in Subect. 2.15.2.

[38] T. Haugset, H. Haugerud, and F. Ravndal, Ann. Phys. 27, 266 (1998).

[39] V. I. Yukalov, Ann. Phys. 323, 461 (2008).

[40] W. H. Dickhoff and D. Van Neck, Many-Body Theory Exposed (World Scientific, 2005).

[41] J. O. Andersen, Rev. Mod. Phys. 76, 599 (2004).

[42] H. Kleinert, EJTP 8, 57 (2011) (klnrt.de/391).

[43] V. I. Yukalov and H. Kleinert, Phys. Rev. A 73, 063612 (2006).

[44] A. Rakhimov, Chul Koo Kim , Sang-Hoon Kim, Jae-Hyung Yee, Phys. Rev. A 77, 033626 (2008).

[45] V. I. Yukalov, Condensed Matter Physics, 16, 23002 (2013).

[46] S. Pilati, S. Giorgini, and N. Prokof'ev, Phys. Rev. Lett. 100, 140405 (2008).

[47] H. Shi and A. Griffin, Phys.Rep. 304, 1 (1998). 